\title{
JACQUET MODULES AND LOCAL LANGLANDS CORRESPONDENCE
}

\author{
HIRAKU ATOBE
}

\begin{abstract}
In this paper, we explicitly compute the semisimplifications of all Jacquet modules of irreducible representations with generic $L$-parameters of $p$-adic split odd special orthogonal groups or symplectic groups. Our computation represents them in terms of linear combinations of standard modules with rational coefficients. The main ingredient of this computation is to apply Mœglin's explicit construction of local $A$-packets to tempered $L$ packets.
\end{abstract}

\section{INTRODUCTION}

When $G$ is a $p$-adic reductive group and $P=M N$ is a parabolic subgroup, there is the normalized induction functor

$$
\operatorname{Ind}_{P}^{G}: \operatorname{Rep}(M) \rightarrow \operatorname{Rep}(G) .
$$

The (normalized) Jacquet functor

$$
\operatorname{Jac}_{P}: \operatorname{Rep}(G) \rightarrow \operatorname{Rep}(M)
$$

is the left adjoint functor of $\operatorname{Ind}_{P}^{G}$. For $\pi \in \operatorname{Rep}(G)$, the object $\operatorname{Jac}_{P}(\pi) \in \operatorname{Rep}(M)$ is called the Jacquet module of $\pi$ with respect to $P$. In the representation theory of $p$-adic reductive groups, the induction functors and the Jacquet functors are ones of the most basic and important terminologies. One of the reasons why they are so important is that they are both exact functors.

The Jacquet modules have many applications. For example:

- Looking at the Jacquet modules of irreducible representation $\pi$ of $G$, one can take a parabolic subgroup $P=M N$ and an irreducible supercuspidal representation $\rho_{M}$ of $M$ such that $\pi \hookrightarrow \operatorname{Ind}_{P}^{G}\left(\rho_{M}\right)$. Such a $\rho_{M}$ is called the cuspidal support of $\pi$.

- Casselman's criterion says that the growth of matrix coefficients of an irreducible representation $\pi$ is determined by exponents of the Jacquet modules of $\pi$.

- Møglin explicitly constructed the local $A$-packets, which are the "local factors of Arthur's global classification", by taking Jacquet functors intelligently.

In this paper, we shall give an explicit description of the semisimplifications of Jacquet modules of tempered representations of split odd special orthogonal groups $\mathrm{SO}_{2 n+1}(F)$ or symplectic groups $\operatorname{Sp}_{2 n}(F)$, where $F$ is a non-archimedean local field of characteristic zero. To do this, it is necessary to have some sort of classification of irreducible representations of these groups. We use the local Langlands correspondence established by Arthur [Ar13] for such a classification.

2010 Mathematics Subject Classification. Primary 22E50; Secondary 11S37.

Key words and phrases. Jacquet module; Local Langlands correspondence. 
The local Langlands correspondence attaches each irreducible representation $\pi$ of $G(F)=$ $\mathrm{SO}_{2 n+1}(F)$ or $G(F)=\mathrm{Sp}_{2 n}(F)$ to its $L$-parameter $(\phi, \eta)$, where

$$
\phi: W_{F} \times \mathrm{SL}_{2}(\mathbb{C}) \rightarrow \mathrm{GL}_{N}(\mathbb{C})
$$

is a self-dual representation of the Weil-Deligne group $W_{F} \times \mathrm{SL}_{2}(\mathbb{C})$ with a suitable structure, and

$$
\eta \in \operatorname{Irr}\left(A_{\phi}\right)
$$

is an irreducible character of the component group $A_{\phi}$ associated to $\phi$ (which is trivial on the central element).

The Jacquet modules will be computed by two main theorems (Theorems 4.2 and 4.3) and Tadić's formula (Theorem 2.7) together with Lemma 2.6. Fix an irreducible unitary supercuspidal representation $\rho$ of $\mathrm{GL}_{d}(F)$. By abuse of notation, we denote by the same notation $\rho$ the irreducible representation of $W_{F}$ corresponding to $\rho$ by the local Langlands correspondence. Let $P_{d}=M_{d} N_{d}$ be the standard parabolic subgroup of $G(F)$ with Levi subgroup $M_{d} \cong \mathrm{GL}_{d}(F) \times G_{0}(F)$ for some classical group $G_{0}$ of the same type as $G$. For an irreducible representation $\pi$, if the semisimplification of $\operatorname{Jac}_{P_{d}}(\pi)$ is of the form

$$
\text { s.s.Jac } \operatorname{Jac}_{d}(\pi)=\bigoplus_{i \in I} \tau_{i} \otimes \pi_{i}
$$

we set

$$
\operatorname{Jac}_{\rho|\cdot| x}(\pi)=\bigoplus_{\substack{i \in I \\ \tau_{i} \cong \rho|\cdot|^{x}}} \pi_{i}
$$

for $x \in \mathbb{R}$. The first main theorem is the description of $\operatorname{Jac}_{\rho|\cdot| x}(\pi)$ for tempered $\pi$ (Theorem 4.2). To state this theorem clearly, we introduce an enhanced component group $\mathcal{A}_{\phi}$ attached to $\phi$ with a canonical surjection $\mathcal{A}_{\phi} \rightarrow A_{\phi}$ in 33.1 .

For discrete series $\pi$, Theorem 4.2 has been proven by Xu [X17a, Lemma 7.3] to describe the cuspidal support of $\pi$ in terms of its $L$-parameter. As related works, Aubert-MoussaouiSolleveld [AMS18, AMSa, AMSb] defined the "cuspidality" of $L$-parameters $(\phi, \eta)$ by a geometric way, and compared this notion with the cuspidal supports or the Bernstein components of corresponding $\pi$. Theorem 4.2 gives us more information for $\pi$ than its cuspidal support. The main ingredient for the proof of Theorem 4.2 is Mœglin's explicit construction of tempered $L$-packets (Theorem 3.3.).

The second main theorem (Theorem 4.3) is a reduction of the computation of the Jacquet module s.s. $\operatorname{Jac}_{P_{k}}(\pi)$ with respect to any maximal parabolic subgroup $P_{k}$ to the one of $\mathrm{Jac}_{\rho|\cdot|{ }^{x}}(\pi)$. Using Theorems 4.2 and 4.3 (together with Lemma 2.6), we can explicitly compute the semisimplifications of all Jacquet modules of irreducible tempered representations $\pi$. In fact, using a generalization of the standard module conjecture by Mœglin-Waldspurger (Theorem 3.2) and Tadić's formula (Theorem 2.7), we can apply this explicit computation to any irreducible representation $\pi$ with generic $L$-parameter $(\phi, \eta)$.

This paper is organized as follows. In 92 , we review some basic results on induced representations and Jacquet modules for classical groups. In particular, Tadić's formula, which computes the Jacquet modules of induced representations, is stated in 2.2 . In 93 , we explain the local Langlands correspondence and Mœglin's explicit construction of tempered $L$-packets. 
In $\S_{4}$, we state the main theorems (Theorems 4.2 and 4.3) and give some examples. Finally, we prove the main theorems in $\$ 5$

Notation. Let $F$ be a non-archimedean local field of characteristic zero. We denote by $W_{F}$ the Weil group of $F$. The norm map $|\cdot|: W_{F} \rightarrow \mathbb{R}^{\times}$is normalized so that $\mid$Frob $\mid=q^{-1}$, where Frob $\in W_{F}$ is a fixed (geometric) Frobenius element, and $q=q_{F}$ is the cardinality of the residual field of $F$.

Each irreducible supercuspidal representation $\rho$ of $\mathrm{GL}_{d}(F)$ is identified with the irreducible bounded representation of $W_{F}$ of dimension $d$ via the local Langlands correspondence for $\mathrm{GL}_{d}$. Through this paper, we fix such a $\rho$. For each positive integer $a$, the unique irreducible algebraic representation of $\mathrm{SL}_{2}(\mathbb{C})$ of dimension $a$ is denoted by $S_{a}$.

For a $p$-adic group $G$, we denote by $\operatorname{Rep}(G)($ resp. $\operatorname{Irr}(G))$ the set of equivalence classes of smooth admissible (resp. irreducible) representations of $G$. For $\Pi \in \operatorname{Rep}(G)$, we write s.s.( $\Pi)$ for the semisimplification of $\Pi$.

\section{IndUCED REPRESENTATIONS AND JACQUET MODULES}

In this section, we recall some results on induced representations and Jacquet modules.

2.1. Representations of $\mathrm{GL}_{k}(F)$. Let $P=M N$ be a standard parabolic subgroup of $\mathrm{GL}_{k}(F)$, i.e., $P$ contains the Borel subgroup consisting of upper half triangular matrices. Then the Levi subgroup $M$ is isomorphic to $\mathrm{GL}_{k_{1}}(F) \times \cdots \times \mathrm{GL}_{k_{r}}(F)$ with $k_{1}+\cdots+k_{r}=k$. For smooth representations $\tau_{1}, \ldots, \tau_{r}$ of $\mathrm{GL}_{k_{1}}(F), \ldots, \mathrm{GL}_{k_{r}}(F)$, respectively, we denote the normalized induced representation by

$$
\tau_{1} \times \cdots \times \tau_{r}:=\operatorname{Ind}_{P}^{\mathrm{GL}_{k}(F)}\left(\tau_{1} \otimes \cdots \otimes \tau_{r}\right) .
$$

A segment is a symbol $[x, y]$, where $x, y \in \mathbb{R}$ with $x-y \in \mathbb{Z}$ and $x \geq y$. We identify $[x, y]$ with the set $\{x, x-1, \ldots, y\}$ so that $\#[x, y]=x-y+1$. Then the normalized induced representation

$$
\rho|\cdot|{ }^{x} \times \cdots \times \rho|\cdot|^{y}
$$

of $\mathrm{GL}_{d(x-y+1)}(F)$ has a unique irreducible subrepresentation, which is denoted by

$$
\langle\rho ; x, \ldots, y\rangle \text {. }
$$

If $y=-x \leq 0$, this is called a Steinberg representation and is denoted by

$$
\operatorname{St}(\rho, 2 x+1)=\langle\rho ; x, \ldots,-x\rangle,
$$

which is a discrete series representation of $\mathrm{GL}_{d(2 x+1)}(F)$. In general, $\langle\rho ; x, \ldots, y\rangle$ is the twist $|\cdot|^{\frac{x+y}{2}} \operatorname{St}(\rho, x-y+1)$. We say that two segments $[x, y]$ and $\left[x^{\prime}, y^{\prime}\right]$ are linked if $[x, y] \not \subset$ $\left[x^{\prime}, y^{\prime}\right],\left[x^{\prime}, y^{\prime}\right] \not \subset[x, y]$ as sets, and $[x, y] \cup\left[x, y^{\prime}\right]$ is also a segment. The linked-ness gives an irreducibility criterion for induced representations.

Theorem 2.1 (Zelevinsky [Z80, Theorem 9.7]). Let $[x, y]$ and $\left[x^{\prime}, y^{\prime}\right]$ be segments, and let $\rho$ and $\rho^{\prime}$ be irreducible unitary supercuspidal representations of $\mathrm{GL}_{d}(F)$ and $\mathrm{GL}_{d^{\prime}}(F)$, respectively. Then the induced representation

$$
\langle\rho ; x, \ldots, y\rangle \times\left\langle\rho^{\prime} ; x^{\prime}, \ldots, y^{\prime}\right\rangle
$$

is irreducible unless $[x, y]$ are $\left[x^{\prime}, y^{\prime}\right]$ are linked, and $\rho \cong \rho^{\prime}$. 
Let $\operatorname{Irr}_{\rho}\left(\mathrm{GL}_{d m}(F)\right)$ be the subset of $\operatorname{Irr}\left(\mathrm{GL}_{d m}(F)\right)$ consisting of $\tau$ with cuspidal support of the form $\rho|\cdot|{ }^{x_{1}} \times \cdots \times \rho|\cdot|^{x_{m}}$, i.e.,

$$
\tau \hookrightarrow \rho|\cdot|{ }^{x_{1}} \times \cdots \times \rho|\cdot|^{x_{m}}
$$

for some $x_{1}, \ldots, x_{m} \in \mathbb{R}$. We understand that $\mathbf{1}:=\mathbf{1}_{\mathrm{GL}_{0}} \in \operatorname{Irr}_{\rho}\left(\mathrm{GL}_{0}(F)\right)$. It is easy to see that

- for pairwise distinct irreducible unitary supercuspidal representations $\rho_{1}, \ldots, \rho_{r}$, if $\tau_{i} \in \operatorname{Irr}_{\rho_{i}}\left(\mathrm{GL}_{d_{i} m_{i}}(F)\right)$ for $i=1, \ldots, r$, then the induced representation $\tau_{1} \times \cdots \times \tau_{r}$ is irreducible;

- any irreducible representation of $\mathrm{GL}_{k}(F)$ is of the above form for some $\tau_{i} \in \operatorname{Irr}_{\rho_{i}}\left(\mathrm{GL}_{d_{i} m_{i}}(F)\right)$.

Lemma 2.2. Let $\Omega_{m}$ be the subset of $\mathbb{R}^{m}$ consisting of elements

$$
\underline{x}=(\underbrace{x_{1}, x_{1}-1, \ldots, y_{1}}_{x_{1}-y_{1}+1}, \underbrace{x_{2}, x_{2}-1, \ldots, y_{2}}_{x_{2}-y_{2}+1}, \ldots, \underbrace{x_{t}, x_{t}-1, \ldots, y_{t}}_{x_{t}-y_{t}+1})
$$

such that $x_{i-1} \leq x_{i}$ for $1<i \leq t$, and $y_{i-1} \leq y_{i}$ if $x_{i-1}=x_{i}$. Let $\Delta_{i}=\left\langle\rho ; x_{i}, x_{i}-1, \ldots, y_{i}\right\rangle$ be the discrete series representation of $\mathrm{GL}_{d\left(x_{i}-y_{i}+1\right)}(F)$ corresponding to the segment $\left[x_{i}, y_{i}\right]$. Then the induced representation $\Delta_{\underline{x}}:=\Delta_{1} \times \cdots \times \Delta_{t}$ has a unique irreducible subrepresentation $\tau_{\underline{x}}$. The map $\underline{x} \mapsto \tau_{\underline{x}}$ gives a bijection

$$
\Omega_{m} \rightarrow \operatorname{Irr}_{\rho}\left(\operatorname{GL}_{d m}(F)\right) .
$$

Proof. This follows from the Langlands classification and Theorem 2.1, See also [Z80, Proposition 9.6].

For a partition $\left(k_{1}, \ldots, k_{r}\right)$ of $k$, we denote by $\operatorname{Jac}_{\left(k_{1}, \ldots, k_{r}\right)}$ the normalized Jacquet functor on $\operatorname{Rep}\left(\mathrm{GL}_{k}(F)\right)$ with respect to the standard maximal parabolic subgroup $P=M N$ with $M \cong \mathrm{GL}_{k_{1}}(F) \times \cdots \times \mathrm{GL}_{k_{r}}(F)$. The Jacquet module of $\langle\rho ; x, \ldots, y\rangle$ with respect to a maximal parabolic subgroup is computed by Zelevinsky.

Proposition 2.3 ([Z80, Proposition 9.5]). Suppose that $x \neq y$ and set $k=d(x-y+1)$. Then $\operatorname{Jac}_{\left(k_{1}, k_{2}\right)}(\langle\rho ; x, \ldots, y\rangle)=0$ unless $k_{1} \equiv 0 \bmod d$. If $k_{1}=d m$ with $1 \leq m \leq x-y$, we have

$$
\operatorname{Jac}_{\left(k_{1}, k_{2}\right)}(\langle\rho ; x, \ldots, y\rangle)=\langle\rho ; x, \ldots, x-(m-1)\rangle \otimes\langle\rho ; x-m, \ldots, y\rangle .
$$

If

for $x \in \mathbb{R}$, we set

$$
\text { s.s. } \operatorname{Jac}_{(d, k-d)}(\tau)=\bigoplus_{i \in I} \tau_{i} \otimes \tau_{i}^{\prime}
$$

$$
\operatorname{Jac}_{\rho|\cdot| x}(\tau)=\bigoplus_{\substack{i \in I \\ \tau_{i} \cong \rho|\cdot|^{x}}} \tau_{i}^{\prime} .
$$

For $\underline{x}=\left(x_{1}, \ldots, x_{r}\right) \in \mathbb{R}^{r}$, we also define

$$
\operatorname{Jac}_{\rho|\cdot| \underline{x}}=\operatorname{Jac}_{\rho|\cdot| x_{r}} \circ \cdots \circ \mathrm{Jac}_{\rho|\cdot|^{x_{1}}} .
$$

This is a functor

$$
\operatorname{Jac}_{\rho|\cdot| \underline{x}}: \operatorname{Rep}\left(\mathrm{GL}_{k}(F)\right) \rightarrow \operatorname{Rep}\left(\mathrm{GL}_{k-d r}(F)\right)
$$

In particular, when $\tau \in \operatorname{Rep}\left(\mathrm{GL}_{d m}(F)\right)$ is of finite length, for $\underline{x}=\left(x_{1}, \ldots, x_{m}\right) \in \mathbb{R}^{m}$, the Jacquet module $\operatorname{Jac}_{\rho|\cdot| \underline{x}}(\tau)$ is a representation of the trivial group $\mathrm{GL}_{0}(F)$ of finite length so that it is a finite dimensional $\mathbb{C}$-vector space. 
Lemma 2.4. Let $\underline{x}=\left(x_{1}, \ldots, y_{1}, \ldots, x_{t}, \ldots, y_{t}\right) \in \Omega_{m}$ such that $x_{i-1} \leq x_{i}$ for $1<i \leq t$, and $y_{i-1} \leq y_{i}$ if $x_{i-1}=x_{i}$ as in Lemma 2.2. For $(x, y) \in\left\{\left(x_{i}, y_{i}\right)\right\}_{i}$, if we set $m_{(x, y)}=$ $\#\left\{i \mid\left(x_{i}, y_{i}\right)=(x, y)\right\}$, then for $\underline{y} \in \Omega_{m}$, we have

$$
\operatorname{dim}_{\mathbb{C}} \operatorname{Jac}_{\rho|\cdot| \underline{y}}\left(\tau_{\underline{x}}\right)= \begin{cases}\prod_{(x, y) \in\left\{\left(x_{i}, y_{i}\right)\right\}_{i}} m_{(x, y)} ! & \text { if } \underline{x^{\prime}}=\underline{x}, \\ 0 & \text { if } \underline{y}<\underline{x} .\end{cases}
$$

Here, we regard $\mathbb{R}^{m}$ as a totally ordered set with respect to the lexicographical order.

Proof. Fix $x \in \mathbb{R}$. We note that $\operatorname{Jac}_{\rho|\cdot| x}\left(\Delta_{\underline{x}}\right) \neq 0$ if and only if $x \in\left\{x_{1}, \ldots, x_{t}\right\}$. Let $\underline{x_{1}^{\prime}}, \ldots, \underline{x_{l}^{\prime}} \in \Omega_{m-1}$ be the elements obtained by removing $x$ from a component of $\underline{x}$, and rearranging it (so that $l=\#\left\{i \mid x_{i}=x\right\}$ ). Then $\operatorname{Jac}_{\rho|\cdot| x}\left(\Delta_{\underline{x}}\right)=\sum_{i=1}^{l} \Delta_{\underline{x_{i}^{\prime}}}$. Using this, we obtain the lemma by induction on $m$.

When $\underline{y}>\underline{x}$, one can also compute $\operatorname{dim}_{\mathbb{C}} \operatorname{Jac}_{\rho|\cdot| \underline{y}}\left(\tau_{\underline{x}}\right)$ inductively.

Let $\mathcal{R}_{k}$ be the Grothendieck group of the category of smooth representations of $\mathrm{GL}_{k}(F)$ of finite length. By the semisimplification, we identify the objects in this category with elements in $\mathcal{R}_{k}$. Elements in $\operatorname{Irr}\left(\mathrm{GL}_{k}(F)\right)$ form a $\mathbb{Z}$-basis of $\mathcal{R}_{k}$. Set $\mathcal{R}=\oplus_{k \geq 0} \mathcal{R}_{k}$. The induction functor gives a product

$$
m: \mathcal{R} \otimes \mathcal{R} \rightarrow \mathcal{R}, \tau_{1} \otimes \tau_{2} \mapsto \text { s.s. }\left(\tau_{1} \times \tau_{2}\right) .
$$

This product makes $\mathcal{R}$ an associative commutative ring. On the other hand, the Jacquet functor gives a coproduct

$$
m^{*}: \mathcal{R} \rightarrow \mathcal{R} \otimes \mathcal{R}
$$

which is defined by the $\mathbb{Z}$-linear extension of

$$
\operatorname{Irr}\left(\mathrm{GL}_{k}(F)\right) \ni \tau \mapsto \sum_{k_{1}=0}^{k} \text { s.s. } \operatorname{Jac}_{\left(k_{1}, k-k_{1}\right)}(\tau) .
$$

Then $m$ and $m^{*}$ make $\mathcal{R}$ a graded Hopf algebra, i.e., $m^{*}: \mathcal{R} \rightarrow \mathcal{R} \otimes \mathcal{R}$ is a ring homomorphism.

2.2. Representations of $\mathrm{SO}_{2 n+1}$ and $\mathrm{Sp}_{2 n}$. We set $G$ to be split $\mathrm{SO}_{2 n+1}$ or $\mathrm{Sp}_{2 n}$, i.e., $G$ is the split algebraic group of type $B_{n}$ or $C_{n}$. Fix a Borel subgroup of $G(F)$, and let $P=M N$ be a standard parabolic subgroup of $G(F)$. Then the Levi part $M$ is of the form $\operatorname{GL}_{k_{1}}(F) \times \cdots \times$ $\mathrm{GL}_{k_{r}}(F) \times G_{0}(F)$ with $G_{0}=\mathrm{SO}_{2 n_{0}+1}$ or $G_{0}=\mathrm{Sp}_{2 n_{0}}$ such that $k_{1}+\cdots+k_{r}+n_{0}=n$. For a smooth representation $\tau_{1} \otimes \cdots \otimes \tau_{r} \otimes \pi_{0}$ of $M$, we denote the normalized induced representation by

$$
\tau_{1} \times \cdots \times \tau_{r} \rtimes \pi_{0}=\operatorname{Ind}_{P}^{G(F)}\left(\tau_{1} \otimes \cdots \otimes \tau_{r} \otimes \pi_{0}\right) .
$$

The functor $\operatorname{Ind}_{P}^{G(F)}: \operatorname{Rep}(M) \rightarrow \operatorname{Rep}(G(F))$ is exact.

On the other hand, for a smooth representation $\pi$ of $G(F)$, we denote the normalized Jacquet module with respect to $P$ by

$$
\operatorname{Jac}_{P}(\pi)
$$

and its semisimplification by s.s.Jac $\operatorname{Jac}_{P}(\pi)$. The functor $\operatorname{Jac}_{P}: \operatorname{Rep}(G(F)) \rightarrow \operatorname{Rep}(M)$ is exact. The Frobenius reciprocity asserts that

$$
\operatorname{Hom}_{G(F)}\left(\pi, \operatorname{Ind}_{P}^{G(F)}(\sigma)\right) \cong \operatorname{Hom}_{M}\left(\operatorname{Jac}_{P}(\pi), \sigma\right)
$$


for $\pi \in \operatorname{Rep}(G(F))$ and $\sigma \in \operatorname{Rep}(M)$.

The maximal parabolic subgroup with Levi $\mathrm{GL}_{k}(F) \times G_{0}(F)$ is denoted by $P_{k}=M_{k} N_{k}$. If

$$
\text { s.s. } \operatorname{Jac}_{P_{d}}(\pi)=\bigoplus_{i \in I} \tau_{i} \otimes \pi_{i}
$$

for a real number $x$, we set

$$
\operatorname{Jac}_{\rho|\cdot| x}(\pi)=\bigoplus_{\substack{i \in I \\ \tau_{i} \cong \rho|\cdot|^{x}}} \pi_{i}
$$

This is a representation of $G_{0}(F)$, which is $\mathrm{SO}_{2(n-d)+1}(F)$ or $\operatorname{Sp}_{2(n-d)}(F)$. Also, for $\underline{x}=$ $\left(x_{1}, \ldots, x_{r}\right) \in \mathbb{R}^{r}$, we set

$$
\operatorname{Jac}_{\rho|\cdot| \underline{x}}(\pi)=\operatorname{Jac}_{\rho|\cdot| x_{1}, \ldots, \rho|\cdot|^{x_{r}}}(\pi)=\operatorname{Jac}_{\rho|\cdot| x_{r}} \circ \cdots \circ \operatorname{Jac}_{\rho|\cdot| x_{1}}(\pi) .
$$

We recall some properties of $\operatorname{Jac}_{\rho|\cdot| x_{1}, \ldots, \rho|\cdot| x_{r}}$.

Lemma 2.5 ([X17a, Lemmas 5.3, 5.6]). Let $\pi$ be an irreducible representation of $G(F)$.

(1) Suppose that $\operatorname{Jac}_{\rho|\cdot|{ }^{x_{1}}, \ldots, \rho|\cdot|{ }^{x_{r}}}(\pi)$ is nonzero. Then there exists an irreducible constituent $\sigma$ of $\operatorname{Jac}_{\rho|\cdot|{ }^{x_{1}}, \ldots, \rho|\cdot| x_{r}}(\pi)$ such that we have an inclusion

$$
\pi \hookrightarrow \rho|\cdot|{ }^{x_{1}} \times \cdots \times \rho|\cdot|^{x_{r}} \rtimes \sigma .
$$

(2) If $|x-y| \neq 1$, then $\operatorname{Jac}_{\left.\rho|\cdot|\right|^{x}, \rho|\cdot| y^{y}}(\pi)=\operatorname{Jac}_{\rho|\cdot| y, \rho|\cdot| x}(\pi)$.

Let $\mathcal{R}_{n}(G)$ be the Grothendieck group of the category of smooth representations of $G(F)$ of finite length, where $n=\operatorname{rank}(G)$, i.e., $G=\mathrm{SO}_{2 n+1}$ or $G=\operatorname{Sp}_{2 n}$. Set $\mathcal{R}(G)=\oplus_{n \geq 0} \mathcal{R}_{n}(G)$. The parabolic induction defines a module structure

$$
\rtimes: \mathcal{R} \otimes \mathcal{R}(G) \rightarrow \mathcal{R}(G), \tau \otimes \pi \mapsto \text { s.s. }(\tau \rtimes \pi),
$$

and the Jacquet functor defines a comodule structure

$$
\mu^{*}: \mathcal{R}(G) \rightarrow \mathcal{R} \otimes \mathcal{R}(G)
$$

by

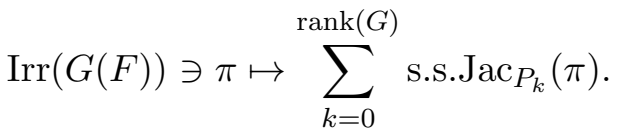

When

$$
\mu^{*}(\pi)=\sum_{i \in I} \tau_{i} \otimes \pi_{i}
$$

we define $\mu_{\rho}^{*}(\pi)$ by

$$
\mu_{\rho}^{*}(\pi)=\sum_{\substack{i \in I \\ \tau_{i} \in \operatorname{Irr}_{\rho}\left(\mathrm{GL}_{d m}(F)\right)}} \tau_{i} \otimes \pi_{i} .
$$

Lemma 2.6. If we define $\iota: \mathcal{R}(G) \rightarrow \mathcal{R} \otimes \mathcal{R}(G)$ by $\pi \mapsto \mathbf{1}_{\mathrm{GL}_{0}(F)} \otimes \pi$, we have

$$
\mu^{*}=\circ_{\rho}\left((m \otimes \mathrm{id}) \circ\left(\mathrm{id} \otimes \mu_{\rho}^{*}\right)\right) \circ \iota,
$$

where $\rho$ runs over all irreducible unitary supercuspidal representations of $\mathrm{GL}_{d}(F)$ for $d>0$. 
Proof. Fix an irreducible representation $\pi$ of $G(F)$. First, we note that there are only finitely many $\rho$ such that $\mu_{\rho}^{*}(\pi) \neq 0$. Second, we claim that

$$
(m \otimes \mathrm{id}) \circ\left(\mathrm{id} \otimes \mu_{\rho^{\prime}}^{*}\right) \circ \mu_{\rho}^{*}(\pi)=(m \otimes \mathrm{id}) \circ\left(\mathrm{id} \otimes \mu_{\rho}^{*}\right) \circ \mu_{\rho^{\prime}}^{*}(\pi)
$$

for distinct $\rho$ and $\rho^{\prime}$. In fact, this is the sum of subrepresentations appearing $\mu^{*}(\pi)$ of the form

$$
\left(\tau \times \tau^{\prime}\right) \otimes \pi_{0}=\left(\tau^{\prime} \times \tau\right) \otimes \pi_{0},
$$

where $\tau$ is of type $\rho$ and $\tau^{\prime}$ is of type $\rho^{\prime}$. By the same argument, we have

$$
\mu^{*}=\circ_{\rho}\left((m \otimes \mathrm{id}) \circ\left(\mathrm{id} \otimes \mu_{\rho}^{*}\right)\right) \circ \iota(\pi),
$$

as desired.

Tadić established a formula to compute $\mu^{*}$ for induced representations. The contragredient functor $\tau \mapsto \tau^{\vee}$ defines an automorphism $\vee: \mathcal{R} \rightarrow \mathcal{R}$ in a natural way. Let $s: \mathcal{R} \otimes \mathcal{R} \rightarrow \mathcal{R} \otimes \mathcal{R}$ be the homomorphism defined by $\sum_{i} \tau_{i} \otimes \tau_{i}^{\prime} \mapsto \sum_{i} \tau_{i}^{\prime} \otimes \tau_{i}$.

Theorem 2.7 (Tadić [T95]). Consider the composition

$$
M^{*}=(m \otimes \mathrm{id}) \circ\left(\vee \otimes m^{*}\right) \circ s \circ m^{*}: \mathcal{R} \rightarrow \mathcal{R} \otimes \mathcal{R} .
$$

Then for the maximal parabolic subgroup $P_{k}=M_{k} N_{k}$ of $G(F)$ and for an admissible representation $\tau \otimes \pi$ of $M_{k}$, we have

$$
\mu^{*}(\tau \rtimes \pi)=M^{*}(\pi) \rtimes \mu^{*}(\pi) .
$$

In particular, we have the following.

Corollary 2.8. For a segment $[x, y]$, we have

$$
\begin{aligned}
& \mu_{\rho}^{*}(\langle\rho ; x, \ldots, y\rangle \rtimes \pi)=\sum_{\substack{k, l \geq 0 \\
k+l \leq x-y+1}} \\
& ((\langle\rho ; \underbrace{-y, \ldots,-y-k+1}_{k}\rangle \times\langle\rho ; \underbrace{x, \ldots, x-l+1}_{l}\rangle) \otimes\langle\rho ; x-l, \ldots, y+k\rangle) \rtimes \mu_{\rho}^{*}(\pi) .
\end{aligned}
$$

\section{LOCAL LANGLANDS CORRESPONDENCE} $F$.

In this section, we review the local Langlands correspondence for split $\mathrm{SO}_{2 n+1}$ or $\mathrm{Sp}_{2 n}$ over

3.1. L-parameters. A homomorphism

$$
\phi: W_{F} \times \mathrm{SL}_{2}(\mathbb{C}) \rightarrow \mathrm{GL}_{k}(\mathbb{C})
$$

is called a representation of the Weil-Deligne group $W_{F} \times \mathrm{SL}_{2}(\mathbb{C})$ if

- $\phi($ Frob $) \in \mathrm{GL}_{k}(\mathbb{C})$ is semisimple;

- $\phi \mid W_{F}$ is smooth, i.e., has an open kernel;

- $\phi \mid \mathrm{SL}_{2}(\mathbb{C})$ is algebraic. 
We say that a representation $\phi$ of $W_{F} \times \mathrm{SL}_{2}(\mathbb{C})$ is tempered if $\phi\left(W_{F}\right)$ is bounded. The local Langlands correspondence for $\mathrm{GL}_{k}$ asserts that there is a canonical bijection

$$
\operatorname{Irr}\left(\mathrm{GL}_{k}(F)\right) \longleftrightarrow\left\{\phi: W_{F} \times \mathrm{SL}_{2}(\mathbb{C}) \rightarrow \mathrm{GL}_{k}(\mathbb{C})\right\} / \cong,
$$

which preserves the tempered-ness.

An $L$-parameter for $\mathrm{SO}_{2 n+1}$ is a symplectic representation

$$
\phi: W_{F} \times \mathrm{SL}_{2}(\mathbb{C}) \rightarrow \mathrm{Sp}_{2 n}(\mathbb{C}) .
$$

Similarly, an $L$-parameter for $\mathrm{Sp}_{2 n}$ is an orthogonal representation

$$
\phi: W_{F} \times \mathrm{SL}_{2}(\mathbb{C}) \rightarrow \mathrm{SO}_{2 n+1}(\mathbb{C}) .
$$

For $G=\mathrm{SO}_{2 n+1}$ or $G=\mathrm{Sp}_{2 n}$, we let $\Phi(G)$ be the set of equivalence classes of $L$-parameters for $G$. We say that

- $\phi \in \Phi(G)$ is discrete if $\phi$ is a multiplicity-free sum of irreducible self-dual representations of the same type as $\phi$;

- $\phi \in \Phi(G)$ is of good parity if $\phi$ is a sum of irreducible self-dual representations of the same type as $\phi$;

- $\phi \in \Phi(G)$ is tempered if $\phi\left(W_{F}\right)$ is bounded;

- $\phi \in \Phi(G)$ is generic if the adjoint $L$-function $L(s, \phi, \mathrm{Ad})$ is regular at $s=1$.

We denote by $\Phi_{\text {disc }}(G)$ (resp. $\Phi_{\text {gp }}(G), \Phi_{\text {temp }}(G)$, and $\Phi_{\text {gen }}(G)$ ) the subset of $\Phi(G)$ consisting of discrete $L$-parameters (resp. $L$-parameters of good parity, tempered $L$-parameters, and generic $L$-parameters). Then we have inclusions

$$
\Phi_{\text {disc }}(G) \subset \Phi_{\text {gp }}(G) \subset \Phi_{\text {temp }}(G) \subset \Phi_{\text {gen }}(G) \subset \Phi(G) .
$$

For $\phi \in \Phi(G)$, we can decompose

$$
\phi=m_{1} \phi_{1} \oplus \cdots \oplus m_{r} \phi_{r} \oplus\left(\phi^{\prime} \oplus \phi^{\prime \vee}\right),
$$

where $\phi_{1}, \ldots, \phi_{r}$ are distinct irreducible self-dual representations of the same type as $\phi, m_{i} \geq 1$ is the multiplicity of $\phi_{i}$ in $\phi$, and $\phi^{\prime}$ is a sum of irreducible representations which are not selfdual or self-dual of the opposite type to $\phi$. We define the component group $A_{\phi}$ of $\phi$ by

$$
A_{\phi}=\bigoplus_{i=1}^{r}(\mathbb{Z} / 2 \mathbb{Z}) \alpha_{\phi_{i}} \cong(\mathbb{Z} / 2 \mathbb{Z})^{r} .
$$

Namely, $A_{\phi}$ is a free $\mathbb{Z} / 2 \mathbb{Z}$-module of rank $r$ and $\left\{\alpha_{\phi_{1}}, \ldots, \alpha_{\phi_{r}}\right\}$ is a basis of $A_{\phi}$ with $\alpha_{\phi_{i}}$ associated to $\phi_{i}$. We set

$$
z_{\phi}=\sum_{i=1}^{r} m_{i} \alpha_{\phi_{i}} \in A_{\phi}
$$

and we call $z_{\phi}$ the central element in $A_{\phi}$.

We shall introduce an enhanced component group $\mathcal{A}_{\phi}$ associated to $\phi \in \Phi(G)$. Write $\phi=\phi_{\mathrm{gp}} \oplus\left(\phi^{\prime} \oplus \phi^{\prime}\right)$, where $\phi_{\mathrm{gp}}$ is the sum of irreducible self-dual representations of the same type as $\phi$, and $\phi^{\prime}$ is a sum of irreducible representations which are not of the same type as $\phi$. We decompose

$$
\phi_{\mathrm{gp}}=\bigoplus_{i \in I} \phi_{i}
$$


into the sum of irreducible representations. Then we define the enhanced component group $\mathcal{A}_{\phi}$ associated to $\phi$ by

$$
\mathcal{A}_{\phi}=\bigoplus_{i \in I}(\mathbb{Z} / 2 \mathbb{Z}) \alpha_{i}
$$

Namely, $\mathcal{A}_{\phi}$ is a free $\mathbb{Z} / 2 \mathbb{Z}$-module whose rank is equal to the length of $\phi_{\mathrm{gp}}$. By abuse of notation, we put $z_{\phi}=\sum_{i \in I} \alpha_{i} \in \mathcal{A}_{\phi}$ and call it the central element in $\mathcal{A}_{\phi}$. There is a canonical surjection

$$
\mathcal{A}_{\phi} \rightarrow A_{\phi}, \alpha_{i} \mapsto \alpha_{\phi_{i}}
$$

The kernel of this map is generated by $\alpha_{i}+\alpha_{j}$ for $\phi_{i} \cong \phi_{j}$. Moreover, this map preserves the central elements.

3.2. Local Langlands correspondence. We denote by $\operatorname{Irr}_{\text {disc }}(G(F))\left(\operatorname{resp} . \operatorname{Irr}_{\text {temp }}(G(F))\right)$ the set of equivalence classes of irreducible discrete series (resp. tempered) representations of $G(F)$. The local Langlands correspondence established by Arthur is as follows:

Theorem 3.1 (실, Theorem 2.2.1]). Let $G$ be a split $\mathrm{SO}_{2 n+1}$ or $\mathrm{Sp}_{2 n}$.

(1) There exists a canonical surjection

$$
\operatorname{Irr}(G(F)) \rightarrow \Phi(G) .
$$

For $\phi \in \Phi(G)$, we denote by $\Pi_{\phi}$ the inverse image of $\phi$ under this map, and call $\Pi_{\phi}$ the L-packet associated to $\phi$.

(2) There exists an injection

$$
\Pi_{\phi} \rightarrow \widehat{A_{\phi}},
$$

which satisfies certain endoscopic character identities. Here, $\widehat{A_{\phi}}$ is the Pontryagin dual of $A_{\phi}$. The image of this map is

$$
\left\{\eta \in \widehat{A_{\phi}} \mid \eta\left(z_{\phi}\right)=1\right\} \text {. }
$$

When $\pi \in \Pi_{\phi}$ corresponds to $\eta \in \widehat{A_{\phi}}$, we write $\pi=\pi(\phi, \eta)$.

(3) For $* \in\{$ disc, temp $\}$,

$$
\operatorname{Irr}_{*}(G(F))=\bigsqcup_{\phi \in \Phi_{*}(G)} \Pi_{\phi} .
$$

(4) Assume that $\phi=\phi_{\tau} \oplus \phi_{0} \oplus \phi_{\tau}^{\vee} \in \Phi_{\text {temp }}(G)$, where

- $\phi_{0} \in \Phi_{\text {temp }}\left(G_{0}\right)$ with a classical group $G_{0}$ of the same type as $G$;

- $\phi_{\tau}$ is a tempered representation of $W_{F} \times \mathrm{SL}_{2}(\mathbb{C})$ of dimension $k$.

Let $\tau$ be the irreducible tempered representation of $\mathrm{GL}_{k}(F)$ corresponding to $\phi_{\tau}$. Then for $\pi_{0} \in \Pi_{\phi_{0}}$, the induced representation $\tau \rtimes \pi_{0}$ decomposes into a direct sum of irreducible tempered representations of $G(F)$. The L-packet $\Pi_{\phi}$ is given by

$$
\Pi_{\phi}=\left\{\pi \mid \pi \subset \tau \rtimes \pi_{0}, \pi_{0} \in \Pi_{\phi_{0}}\right\} .
$$

Moreover there is a canonical inclusion $A_{\phi_{0}} \hookrightarrow A_{\phi}$. If $\pi(\phi, \eta)$ is a direct summand of $\tau \rtimes \pi_{0}$ with $\pi_{0}=\pi\left(\phi_{0}, \eta_{0}\right)$, then $\eta_{0}=\eta \mid A_{\phi_{0}}$.

(5) Assume that

$$
\phi=\phi_{1}|\cdot|^{s_{1}} \oplus \cdots \oplus \phi_{r}|\cdot|^{s_{r}} \oplus \phi_{0} \oplus \phi_{r}^{\vee}|\cdot|^{-s_{r}} \oplus \cdots \oplus \phi_{1}^{\vee}|\cdot|^{-s_{1}},
$$

where

- $\phi_{0} \in \Phi_{\text {temp }}\left(G_{0}\right)$ with a classical group $G_{0}$ of the same type as $G$; 
- $\phi_{i}$ is a tempered representation of $W_{F} \times \mathrm{SL}_{2}(\mathbb{C})$ of dimension $k_{i}$ for $1 \leq i \leq r$;

- $s_{i}$ is a real number such that $s_{1} \geq \cdots \geq s_{r}>0$.

Let $\tau_{i}$ be the irreducible tempered representation of $\mathrm{GL}_{k_{i}}(F)$ corresponding to $\phi_{i}$. Then the L-packet $\Pi_{\phi}$ consists of the unique irreducible quotients $\pi$ of the standard modules

$$
\tau_{1}|\cdot|^{s_{1}} \times \cdots \times \tau_{r}|\cdot|^{s_{r}} \rtimes \pi_{0},
$$

where $\pi_{0}$ runs over $\Pi_{\phi_{0}}$. Moreover there is a canonical inclusion $A_{\phi_{0}} \hookrightarrow A_{\phi}$, which is in fact bijective. If $\pi(\phi, \eta)$ is the unique irreducible quotient of the above standard module with $\pi_{0}=\pi\left(\phi_{0}, \eta_{0}\right)$, then $\eta_{0}=\eta \mid A_{\phi_{0}}$. In this case, we denote this standard module by $I(\phi, \eta)$.

The injection $\Pi_{\phi} \rightarrow \widehat{A_{\phi}}$ is not canonical when $G=\mathrm{Sp}_{2 n}$. To specify this, we implicitly fix an $F^{\times 2}$-orbit of non-trivial additive characters of $F$ through this paper.

We have the following irreducibility criterion for standard modules.

Theorem 3.2 (Generalized standard module conjecture). For $\phi \in \Phi(G)$, the standard module $I(\phi, \mathbf{1})$ attached to $\pi(\phi, \mathbf{1})$ is irreducible if and only if $\phi$ is generic. Moreover, if $\phi$ is generic, then all standard modules $I(\phi, \eta)$, where $\eta \in \widehat{A_{\phi}}$ with $\eta\left(z_{\phi}\right)=1$, are irreducible.

Proof. The first assertion is the usual standard module conjecture proven in CS98, Mu01, HM07, HO13]. The second assertion was proven by Møglin-Waldspurger [MW12, Corollaire 2.14] for special orthogonal groups and symplectic groups. Heiermann [H16] also proved the second assertion in a more general setting. Note that their definition of generic $L$-parameters might look different from ours. The equivalence of two definitions is called a conjecture of Gross-Prasad and Rallis, which was proven by Gan-Ichino [GI16, Proposition B.1].

However, even if $\phi$ is not generic, there might exist an irreducible standard module $I(\phi, \eta)$. An example of such standard modules will be given by Corollary 5.6 and Example 5.7 below.

3.3. Extension to enhanced component groups. To describe Jacquet modules of $\pi(\phi, \eta)$ for $\phi \in \Phi_{\mathrm{gp}}(G)$, it is useful to extend $\pi(\phi, \eta)$ to the case where $\eta$ is a character of the enhanced component group $\mathcal{A}_{\phi}$ as follows. Recall that there exists a canonical surjection $\mathcal{A}_{\phi} \rightarrow A_{\phi}$ so that we have an injection $\widehat{A_{\phi}} \hookrightarrow \widehat{\mathcal{A}_{\phi}}$. For $\eta \in \widehat{\mathcal{A}_{\phi}}$, set

$$
\pi(\phi, \eta)= \begin{cases}\pi(\phi, \eta) & \text { if } \eta \in \widehat{A_{\phi}}, \eta\left(z_{\phi}\right)=1, \\ 0 & \text { otherwise }\end{cases}
$$

In particular, $\pi(\phi, \eta)$ is irreducible or zero for any $\eta \in \widehat{\mathcal{A}_{\phi}}$.

3.4. Moglin's construction of tempered $L$-packets. The $L$-packets are used for local classification. On the other hand, Arthur Ar13, Theorem 2.2.1] introduced the notion of $A$ packets for global classification. Mœglin constructed the local $A$-packets in her consecutive works (e.g., [M06, M09], etc.). For a detailed why Mœglin's local $A$-packets agree with Arthur's, one can see Xu's paper [X17b] in addition to the original papers of Møglin. Since the tempered $A$-packets are the same notion as the tempered $L$-packets, Mœglin's construction can be applied to the tempered $L$-packets. 
We explain Mœglin's construction of $\Pi_{\phi}$ for $\phi \in \Phi_{\mathrm{gp}}(G)$. Write

$$
\phi=\left(\bigoplus_{i=1}^{t} \rho \otimes S_{a_{i}}\right) \oplus \phi_{e}
$$

with $a_{1} \leq \cdots \leq a_{t}$ and $\rho \otimes S_{a} \not \subset \phi_{e}$ for any $a>0$. Take a new $L$-parameter

$$
\phi_{\gg}=\left(\bigoplus_{i=1}^{t} \rho \otimes S_{a_{i}^{\prime}}\right) \oplus \phi_{e}
$$

for a bigger group $G^{\prime}$ of the same type as $G$ such that

- $a_{1}^{\prime}<\cdots<a_{t}^{\prime}$

- $a_{i}^{\prime} \geq a_{i}$ and $a_{i}^{\prime} \equiv a_{i} \bmod 2$ for any $i$;

Then we can identify $\mathcal{A}_{\phi \gg}$ with $\mathcal{A}_{\phi}$ canonically, i.e., $\mathcal{A}_{\phi \gg}=\mathcal{A}_{\phi}$ and if $\mathcal{A}_{\phi \gg} \ni \alpha \mapsto \alpha_{\rho \bigotimes S_{a^{\prime}}} \in$ $A_{\phi \gg}$, then $\mathcal{A}_{\phi} \ni \alpha \mapsto \alpha_{\rho \bigotimes S_{a_{i}}} \in A_{\phi}$, Let $\eta_{\gg} \in \widehat{\mathcal{A}_{\phi \gg}}$ be the character corresponding to $\eta \in \widehat{\mathcal{A}_{\phi}}$, i.e., $\eta_{\gg}=\eta$ via $\mathcal{A}_{\phi \gg}=\mathcal{A}_{\phi}$.

Theorem 3.3 (Møglin). With the notation above, we have

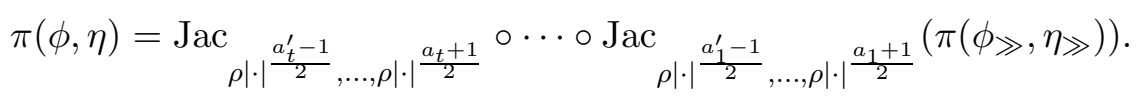

Using this theorem repeatedly, we can construct $L$-packets $\Pi_{\phi}$ for $\phi \in \Phi_{\mathrm{gp}}(G)$ from $L$ packets associated to discrete $L$-parameters for bigger groups..

Example 3.4. We construct $\Pi_{\phi}$ for $\phi=S_{2} \oplus S_{4} \oplus S_{4} \oplus S_{6} \oplus S_{6} \in \Phi_{\mathrm{gp}}\left(\mathrm{SO}_{23}\right)$. Note that $A_{\phi}=(\mathbb{Z} / 2 \mathbb{Z}) \alpha_{S_{2}} \oplus(\mathbb{Z} / 2 \mathbb{Z}) \alpha_{S_{4}} \oplus(\mathbb{Z} / 2 \mathbb{Z}) \alpha_{S_{6}}$ with $z_{\phi}=\alpha_{S_{2}}$. Let $\eta \in \widehat{A_{\phi}}$. We write $\eta\left(\alpha_{S_{2 a}}\right)=$ $\eta_{a} \in\{ \pm 1\}$. If $\eta\left(z_{\phi}\right)=1$, then $\eta_{1}=+1$.

Now we take new L-parameters

$$
\begin{aligned}
\phi_{\gg} & =S_{2} \oplus S_{4} \oplus S_{6} \oplus S_{8} \oplus S_{10} \in \Phi_{\text {disc }}\left(\mathrm{SO}_{31}\right), \\
\phi^{\prime} & =S_{2} \oplus S_{4} \oplus S_{4} \oplus S_{8} \oplus S_{10} \in \Phi_{\text {disc }}\left(\mathrm{SO}_{29}\right), \\
\phi^{\prime \prime} & =S_{2} \oplus S_{4} \oplus S_{4} \oplus S_{6} \oplus S_{10} \in \Phi_{\text {disc }}\left(\mathrm{SO}_{27}\right),
\end{aligned}
$$

and we consider $\eta_{\gg} \in \widehat{A_{\phi \gg}}, \eta^{\prime} \in \widehat{A_{\phi^{\prime}}}$ and $\eta^{\prime \prime} \in \widehat{A_{\phi^{\prime \prime}}}$ given by

- $\eta_{\gg}\left(\alpha_{S_{2}}\right)=\eta^{\prime}\left(\alpha_{S_{2}}\right)=\eta^{\prime \prime}\left(\alpha_{S_{2}}\right)=\eta_{1}=+1$;

- $\eta_{\gg}\left(\alpha_{S_{4}}\right)=\eta_{\gg}\left(\alpha_{S_{6}}\right)=\eta^{\prime}\left(\alpha_{S_{4}}\right)=\eta^{\prime \prime}\left(\alpha_{S_{4}}\right)=\eta_{2}$;

- $\eta_{\gg}\left(\alpha_{S_{8}}\right)=\eta_{\gg}\left(\alpha_{S_{10}}\right)=\eta^{\prime}\left(\alpha_{S_{8}}\right)=\eta^{\prime}\left(\alpha_{S_{10}}\right)=\eta^{\prime \prime}\left(\alpha_{S_{6}}\right)=\eta^{\prime \prime}\left(\alpha_{S_{10}}\right)=\eta_{3}$.

Then Theorem 3.3 says that

$$
\begin{aligned}
\mathrm{Jac}_{\left.|\cdot|\right|^{\frac{5}{2}}}\left(\pi\left(\phi_{\gg}, \eta \gg\right)\right) & =\pi\left(\phi^{\prime}, \eta^{\prime}\right), \\
\operatorname{Jac}_{|\cdot|^{\frac{7}{2}}}\left(\pi\left(\phi^{\prime}, \eta^{\prime}\right)\right) & =\pi\left(\phi^{\prime \prime}, \eta^{\prime \prime}\right), \\
\mathrm{Jac}_{|\cdot| \frac{9}{2},|\cdot|^{\frac{7}{2}}}\left(\pi\left(\phi^{\prime \prime}, \eta^{\prime \prime}\right)\right) & =\pi(\phi, \eta) .
\end{aligned}
$$

\section{Description of Jacquet modules}

In this section, we state the main theorems, which compute the semisimplifications of the Jacquet modules of $\pi(\phi, \eta)$ for $\phi \in \Phi_{\mathrm{gp}}(G)$. 
4.1. Statements. Note that:

Lemma 4.1. For $\phi \in \Phi_{\mathrm{gp}}(G)$ and $x \in \mathbb{R}$, if $\operatorname{Jac}_{\rho|\cdot| x}(\pi) \neq 0$ for some $\pi \in \Pi_{\phi}$, then $x$ is a non-negative half-integer and $\rho \bigotimes S_{2 x+1} \subset \phi$.

Proof. When $\phi \in \Phi_{\text {disc }}(G)$, it follows from [X17a, Lemma 7.3]. We may assume that $\phi \in$ $\Phi_{\mathrm{gp}}(G) \backslash \Phi_{\mathrm{disc}}(G)$. Then there exists an irreducible representation $\rho^{\prime} \otimes S_{a}$ which $\phi$ contains at least multiplicity two. By Theorem 3.1 (4), we have

$$
\pi \hookrightarrow \operatorname{St}\left(\rho^{\prime}, a\right) \rtimes \pi_{0}
$$

for some $\pi_{0} \in \Pi_{\phi_{0}}$ with $\phi_{0}=\phi-\left(\rho^{\prime} \otimes S_{a}\right)^{\oplus 2}$. Then by Corollary 2.8, we have

$$
\begin{aligned}
\operatorname{Jac}_{\rho|\cdot| x}(\pi) & \hookrightarrow\left(1 \otimes \operatorname{St}\left(\rho^{\prime}, a\right)\right) \rtimes \operatorname{Jac}_{\rho|\cdot| x}\left(\pi_{0}\right) \\
& + \begin{cases}2\langle\rho ; x, \ldots,-(x-1)\rangle \rtimes \pi_{0} & \text { if } \rho^{\prime} \cong \rho, a=2 x+1, \\
0 & \text { otherwise. }\end{cases}
\end{aligned}
$$

Hence if $\operatorname{Jac}_{\rho|\cdot| x}(\pi) \neq 0$, then $\operatorname{Jac}_{\rho|\cdot| x}\left(\pi_{0}\right) \neq 0$ or $\rho \otimes S_{2 x+1} \cong \rho^{\prime} \otimes S_{a} \subset \phi$. By induction, we conclude that $\rho \otimes S_{2 x+1} \subset \phi$ as desired.

The following is the first main theorem, which is a description of $\operatorname{Jac}_{\rho|\cdot| x}(\pi(\phi, \eta))$.

Theorem 4.2. Let $\phi \in \Phi_{\mathrm{gp}}(G)$ and $\eta \in \widehat{\mathcal{A}_{\phi}}$ such that $\pi(\phi, \eta) \neq 0$. Fix a non-negative half-integer $x \in(1 / 2) \mathbb{Z}$. Write

$$
\phi=\phi_{0} \oplus\left(\rho \otimes S_{2 x+1}\right)^{\oplus m}
$$

with $\rho \otimes S_{2 x+1} \not \subset \phi_{0}$ and $m>0$.

(1) Assume that $m \geq 3$. Take $\delta \in\{1,2\}$ such that $\delta \equiv m \bmod 2$. Then

$$
\begin{aligned}
& \operatorname{Jac}_{\rho|\cdot|^{x}}(\pi(\phi, \eta)) \\
& =(m-\delta) \cdot\langle\rho ; \underbrace{x, x-1, \ldots,-(x-1)}_{2 x}\rangle \rtimes \pi\left(\phi-\left(\rho \otimes S_{2 x+1}\right)^{\oplus 2}, \eta\right) \\
& +\underbrace{\operatorname{St}(\rho, 2 x+1) \times \cdots \times \operatorname{St}(\rho, 2 x+1)}_{(m-\delta) / 2} \rtimes \operatorname{Jac}_{\rho|\cdot| x}\left(\pi\left(\phi_{0} \oplus\left(\rho \otimes S_{2 x+1}\right)^{\oplus \delta}, \eta\right)\right) .
\end{aligned}
$$

Here, we canonically identify the (usual) component groups of $\phi-\left(\rho \otimes S_{2 x+1}\right)^{\oplus 2}$ and $\phi_{0} \oplus\left(\rho \otimes S_{2 x+1}\right)^{\oplus \delta}$ with $A_{\phi}$, so that we regard $\eta$ as a character of these groups.

(2) Assume that $x>0$ and $m=1$. Set

$$
\phi^{\prime}=\phi-\left(\rho \otimes S_{2 x+1}\right) \oplus\left(\rho \otimes S_{2 x-1}\right) .
$$

There is a canonical inclusion $\mathcal{A}_{\phi^{\prime}} \hookrightarrow \mathcal{A}_{\phi}$, which is in fact bijective if $x>1 / 2$. Let $\eta^{\prime} \in \widehat{\mathcal{A}_{\phi^{\prime}}}$ be the character corresponding to $\eta \in \widehat{\mathcal{A}_{\phi}}$, i.e., $\eta^{\prime}=\eta \mid \mathcal{A}_{\phi^{\prime}}$. Then

$$
\operatorname{Jac}_{\rho|\cdot| x}(\pi(\phi, \eta))=\pi\left(\phi^{\prime}, \eta^{\prime}\right) .
$$

In particular, $\operatorname{Jac}_{\rho|\cdot| x}(\pi(\phi, \eta))$ is irreducible or zero. 
(3) Assume that $x>0$ and $m=2$. Set $\eta_{+}=\eta$, and take the unique character $\eta_{-} \in \widehat{\mathcal{A}_{\phi}}$ so that $\eta_{-}\left|\mathcal{A}_{\phi_{0}}=\eta_{+}\right| \mathcal{A}_{\phi_{0}}, \eta_{-}\left(z_{\phi}\right)=\eta_{+}\left(z_{\phi}\right)=1$ but $\eta_{-} \neq \eta_{+}$. For $\phi^{\prime}$ as in (3) and for $\epsilon \in\{ \pm 1\}$, let $\eta_{\epsilon}^{\prime} \in \widehat{\mathcal{A}_{\phi^{\prime}}}$ be the character corresponding to $\eta_{\epsilon} \in \widehat{\mathcal{A}_{\phi}}$ via the canonical inclusion $\mathcal{A}_{\phi^{\prime}} \hookrightarrow \mathcal{A}_{\phi}$. Then

$\operatorname{Jac}_{\rho|\cdot| x}(\pi(\phi, \eta))=\langle\rho ; x, x-1, \ldots,-(x-1)\rangle \rtimes \pi\left(\phi_{0}, \eta \mid \mathcal{A}_{\phi_{0}}\right)+\pi\left(\phi^{\prime}, \eta_{+}^{\prime}\right)-\pi\left(\phi^{\prime}, \eta_{-}^{\prime}\right)$.

(4) Assume that $x=0$. If $m=1$, then $\operatorname{Jac}_{\rho}(\pi(\phi, \eta))=0$. If $m=2$, then $\operatorname{Jac}_{\rho}(\pi(\phi, \eta))=$ $\pi\left(\phi_{0}, \eta \mid \mathcal{A}_{\phi_{0}}\right)$.

When $\phi \in \Phi_{\text {disc }}(G)$, Theorem 4.2 has been already proven by Xu ([X17a, Lemma 7.3]). In (2) (resp. (3)), we note that $\pi\left(\phi^{\prime}, \eta^{\prime}\right)$ (resp. $\left.\pi\left(\phi^{\prime}, \eta_{\epsilon}^{\prime}\right)\right)$ can be zero even if $\pi(\phi, \eta) \neq 0$. In (3), the character $\eta_{-}$is characterized so that

$$
\pi\left(\phi, \eta_{+}\right) \oplus \pi\left(\phi, \eta_{-}\right)=\operatorname{St}(\rho, 2 x+1) \rtimes \pi\left(\phi_{0}, \eta \mid \mathcal{A}_{\phi_{0}}\right) .
$$

The second main theorem concerns $\mu_{\rho}^{*}(\pi)$.

Theorem 4.3. Let $\phi \in \Phi_{\mathrm{gp}}(G)$, and write

$$
\phi=\left(\bigoplus_{i=1}^{t} \rho \otimes S_{a_{i}}\right) \oplus \phi_{e}
$$

with $a_{1} \leq \cdots \leq a_{t}$ and $\rho \otimes S_{a} \not \subset \phi_{e}$ for any $a>0$, and $a_{i}=2 x_{i}+1$. For $0 \leq m \leq$ $(2 d)^{-1} \cdot \operatorname{dim}(\phi)$, we denote by $K_{\phi}^{(m)}$ the set of tuples of integers $\underline{k}=\left(k_{1}, \ldots, k_{t}\right)$ such that

- $0 \leq k_{i} \leq a_{i}$ for any $i$;

- $k_{i-1} \geq k_{i}$ if $a_{i-1}=a_{i}$;

- $k_{1}+\cdots+k_{t}=m$.

For $\underline{k} \in K_{\phi}^{(m)}$, set

$$
\underline{x}(\underline{k})=(\underbrace{x_{1}, \ldots, x_{1}-k_{1}+1}_{k_{1}}, \ldots, \underbrace{x_{t}, \ldots, x_{t}-k_{t}+1}_{k_{t}}) \in \Omega_{m} .
$$

For $\underline{k}, \underline{l} \in K_{\phi}$, we set

$$
m_{\underline{k}, \underline{l}}=\operatorname{dim}_{\mathbb{C}} \operatorname{Jac}_{\rho|\cdot| \underline{x}(\underline{k})}\left(\Delta_{\underline{x}(\underline{l})}\right),
$$

and define $\left(m_{\underline{k}, \underline{l}}^{\prime}\right)_{\underline{k}, \underline{l} \in K_{\phi}^{(m)}}$ to be the inverse matrix of $\left(m_{\underline{k}, \underline{l}}\right)_{\underline{k}, \underline{l} \in K_{\phi}^{(m)}}$, i.e.,

$$
\sum_{\underline{k^{\prime}} \in K_{\phi}^{(m)}} m_{\underline{k}, \underline{k^{\prime}}}^{\prime} m_{\underline{k^{\prime}, \underline{l}}}= \begin{cases}1 & \text { if } \underline{k}=\underline{l}, \\ 0 & \text { if } \underline{k} \neq \underline{l} .\end{cases}
$$

Then for $\pi \in \Pi_{\phi}$, we have

$$
\mu_{\rho}^{*}(\pi)=\sum_{m=0}^{(2 d)^{-1} \operatorname{dim}(\phi)} \sum_{\underline{k}, \underline{l} \in K_{\phi}^{(m)}} m_{\underline{k}, \underline{l}}^{\prime} \cdot \Delta_{\underline{x}(\underline{k})} \otimes \operatorname{Jac}_{\rho|\cdot| \underline{x}(\underline{l})}(\pi) .
$$

When we formally regard $\left(\Delta_{\underline{x}(\underline{k})}\right)_{\underline{k} \in K_{\phi}^{(m)}}$ and $\left(\otimes \operatorname{Jac}_{\rho|\cdot| \underline{x}(\underline{l})}(\pi)\right)_{\underline{l} \in K_{\phi}^{(m)}}$ as column vectors, we have

$$
\sum_{\underline{k}, \underline{l} \in K_{\phi}^{(m)}} m_{\underline{k}, \underline{l}}^{\prime} \cdot \Delta_{\underline{x}(\underline{k})} \otimes \operatorname{Jac}_{\rho|\cdot| \underline{x}(\underline{l})}(\pi)={ }^{t}\left(\Delta_{\underline{x}(\underline{k})}\right) \cdot\left(m_{\underline{k}, \underline{l}}\right)^{-1} \cdot\left(\otimes \operatorname{Jac}_{\rho|\cdot| \underline{x}(\underline{l})}(\pi)\right) .
$$


By Lemma 2.4. $\left(m_{\underline{k}, \underline{l}}\right)_{\underline{k}, \underline{l} \in K_{\phi}^{(m)}}$ is a "triangular matrix", which can be computed inductively. Here, we regard $K_{\phi}^{(m)}$ as a totally ordered set with respect to the lexicographical order. The diagonal entries $m_{\underline{k}, \underline{k}}$ are given in Lemma 2.4 explicitly.

By Tadić's formula (Theorem 2.7), Lemma 2.6, and Theorems 4.2, 4.3, we can deduce the following corollary.

Corollary 4.4. We can compute $\mu^{*}(\pi)$ explicitly for any $\pi \in \Pi_{\phi}$ with $\phi \in \Phi_{\text {gen }}(G)$.

4.2. Examples. We shall give some examples.

Example 4.5. Fix two positive integers $a, b$ such that $a \equiv b \bmod 2$ and $a<b$, and consider

$$
\phi=\rho \bigotimes\left(S_{a} \oplus S_{b}\right) \in \Phi_{\mathrm{disc}}\left(\mathrm{SO}_{d(a+b)+1}\right) .
$$

Then $\Pi_{\phi}=\left\{\pi_{+}(a, b), \pi_{-}(a, b)\right\}$ with generic $\pi_{+}(a, b)$ and non-generic $\pi_{-}(a, b)$. Note that both $\pi_{+}(a, b)$ and $\pi_{-}(a, b)$ are discrete series. We compute $\mu^{*}\left(\pi_{\epsilon}(a, b)\right)$ for $\epsilon \in\{ \pm 1\}$.

Note that $K_{\phi}^{(m)}=\left\{\left(k_{1}, k_{2}\right) \in \mathbb{Z}^{2} \mid 0 \leq k_{1} \leq a, 0 \leq k_{2} \leq b, k_{1}+k_{2}=m\right\}$. For $\left(k_{1}, k_{2}\right) \in K_{\phi}^{(m)}$,

$$
\Delta_{\underline{x}\left(k_{1}, k_{1}\right)}=\left\langle\rho ; \frac{a-1}{2}, \ldots, \frac{a+1}{2}-k_{1}\right\rangle \times\left\langle\rho ; \frac{b-1}{2}, \ldots, \frac{b+1}{2}-k_{2}\right\rangle .
$$

This induced representation is irreducible unless $(a+3) / 2-k_{1} \leq(b+1) / 2-k_{2} \leq(a+1) / 2$, i.e., $(b-a) / 2 \leq k_{2} \leq(b-a) / 2+k_{1}-1$. Moreover, one can easy to see that for $\left(l_{1}, l_{2}\right)$, the virtual representation

$$
\sum_{\left(k_{1}, k_{2}\right) \in K_{\phi}^{(m)}} m_{\left(k_{1}, k_{2}\right),\left(l_{1}, l_{2}\right)}^{\prime} \cdot \Delta_{\underline{x}\left(k_{1}, k_{2}\right)}
$$

is the unique irreducible subrepresentation $\tau_{\underline{x}\left(l_{1}, l_{2}\right)}$ of $\Delta_{\underline{x}\left(l_{1}, l_{2}\right)}$ (cf. see [Z80, Proposition 4.6]).

Note that

$$
\mathrm{Jac}_{\left.\rho|\cdot|\right|^{\frac{a-1}{2}}, \ldots, \rho|\cdot|^{\frac{a+1}{2}-k_{1}}}\left(\pi_{ \pm}(a, b)\right)= \begin{cases}\pi_{\epsilon}\left(a-2 k_{1}, b\right) & \text { if } k_{1} \leq a / 2 \\ 0 & \text { otherwise }\end{cases}
$$

Here, when $k_{1}=a / 2$, we understand that $\pi_{+}(0, b)$ is the unique element in $\Pi_{\rho \bowtie S_{b}}$, and $\pi_{-}(0, b)=0$. Moreover, for $\left(k_{1}, k_{2}\right) \in K_{\phi}^{(m)}$, when $k_{1} \leq a / 2$ and $k_{2} \leq(b-a) / 2+k_{1}$, we have

$$
\operatorname{Jac}_{\rho|\cdot| \underline{x}\left(k_{1}, k_{2}\right)}\left(\pi_{\epsilon}(a, b)\right)=\pi_{\epsilon}\left(a-2 k_{1}, b-2 k_{2}\right) .
$$

When $k_{1} \leq a / 2$ and $(b-a) / 2+k_{1}+1 \leq k_{2} \leq b / 2$, we have

$$
\begin{aligned}
\operatorname{Jac}_{\rho|\cdot| \underline{x}\left(k_{1}, k_{2}\right)}\left(\pi_{\epsilon}(a, b)\right)= & \left\langle\rho ; \frac{a-1}{2}-k_{1}, \ldots,-\frac{b-1}{2}+k_{2}\right\rangle \rtimes \mathbf{1}_{\mathrm{SO}_{1}(F)} \\
& +\pi_{\epsilon}\left(b-2 k_{2}, a-2 k_{1}\right)-\pi_{-\epsilon}\left(b-2 k_{2}, a-2 k_{1}\right) .
\end{aligned}
$$

When $k_{1} \leq a / 2$ and $b / 2<k_{2} \leq(a+b) / 2-k_{1}$, we have

$$
\operatorname{Jac}_{\rho|\cdot| \underline{x}\left(k_{1}, k_{2}\right)}\left(\pi_{\epsilon}(a, b)\right)=\left\langle\rho ; \frac{a-1}{2}-k_{1}, \ldots,-\frac{b-1}{2}+k_{2}\right\rangle \rtimes \mathbf{1}_{\mathrm{SO}_{1}(F)} .
$$


In particular, if $k_{1}+k_{2}=(a+b) / 2$, then $\mathrm{Jac}_{\rho|\cdot| \underline{x}\left(k_{1}, k_{2}\right)}\left(\pi_{\epsilon}(a, b)\right)=\mathbf{1}_{\mathrm{SO}_{1}(F)}$. Hence s.s.Jac $\operatorname{Jac}_{P_{d(a+b) / 2}}\left(\pi_{\epsilon}(a, b)\right)$ contains the irreducible representation

$$
\left\langle\rho ; \frac{a-1}{2}, \ldots, \frac{a+1}{2}-k_{1}\right\rangle \times\left\langle\rho ; \frac{b-1}{2}, \ldots,-\left(\frac{a-1}{2}-k_{1}\right)\right\rangle \otimes \mathbf{1}_{\mathrm{SO}_{1}(F)}
$$

with multiplicity one if $k_{1}<a / 2$, or if $k_{1}=a / 2$ and $\epsilon=+1$.

Example 4.6. Consider the L-parameter $\phi=S_{2} \oplus S_{4} \oplus S_{4} \in \Phi_{\mathrm{gp}}\left(\mathrm{SO}_{11}\right)$. Then $\Pi_{\phi}$ has two elements $\pi_{+}(2,4,4)$ and $\pi_{-}(2,4,4)$ with generic $\pi_{+}(2,4,4)$ and non-generic $\pi_{-}(2,4,4)$. Then

$$
K^{(m)}=\left\{\left(k_{1}, k_{2}, k_{3}\right) \in \mathbb{Z}^{3} \mid 0 \leq k_{1} \leq 2,0 \leq k_{3} \leq k_{2} \leq 4, k_{1}+k_{2}+k_{3}=m\right\}
$$

for $0 \leq m \leq 5$. Write $\Pi_{\underline{x}(\underline{k})}^{\epsilon}=\operatorname{Jac}_{\rho|\cdot| \underline{x}(\underline{k})}\left(\pi_{\epsilon}(2,4,4)\right)$ for $\epsilon \in\{ \pm 1\}$, and $\operatorname{St}_{a}=\operatorname{St}\left(\mathbf{1}_{\mathrm{GL}_{1}(F)}, a\right)$.

We denote by $\operatorname{det}_{a}$ the determinant character of $\mathrm{GL}_{a}(F)$.

(1) When $m=1$, we have $K_{\phi}^{(1)}=\{(1,0,0)>(0,1,0)\}$. Since

$$
\left(\Delta_{\underline{x}(1,0,0)} \quad \Delta_{\underline{x}(0,1,0)}\right)=\left(|\cdot|^{\frac{1}{2}}|\cdot|^{\frac{3}{2}}\right),
$$

we have

$$
\left(m_{\underline{k}, \underline{k}^{\prime}}\right)_{\underline{k}, \underline{k^{\prime}}}^{-1}=\left(\begin{array}{ll}
1 & 0 \\
0 & 1
\end{array}\right)^{-1}=\left(\begin{array}{ll}
1 & 0 \\
0 & 1
\end{array}\right)
$$

Moreover

$$
\left(\begin{array}{c}
\Pi_{\underline{x}(1,0,0)}^{\epsilon} \\
\Pi_{\underline{x}(0,1,0)}^{\epsilon}
\end{array}\right)=\left(\begin{array}{c}
\pi_{\epsilon}(4,4) \\
|\cdot| \frac{1}{2} \mathrm{St}_{3} \rtimes \pi_{+}(2)+\epsilon \cdot \pi_{+}(2,2,4)
\end{array}\right) .
$$

Hence

$$
\begin{aligned}
\text { S.s. } \operatorname{Jac}_{P_{1}}\left(\pi_{\epsilon}(2,4,4)\right) & =|\cdot|^{\frac{1}{2}} \otimes \pi_{\epsilon}(4,4) \\
& +|\cdot|^{\frac{3}{2}} \otimes\left(|\cdot|^{\frac{1}{2}} \mathrm{St}_{3} \rtimes \pi_{+}(2)+\epsilon \cdot \pi_{+}(2,2,4)\right) .
\end{aligned}
$$

(2) When $m=2$, we have $K_{\phi}^{(2)}=\{(2,0,0)>(1,1,0)>(0,2,0)>(0,1,1)\}$. Since

$$
\begin{aligned}
& \left(\begin{array}{llll}
\Delta_{\underline{x}(2,0,0)} & \Delta_{\underline{x}(1,1,0)} & \Delta_{\underline{x}(0,2,0)} & \Delta_{\underline{x}(0,1,1)}
\end{array}\right) \\
& =\left(\begin{array}{llll}
\mathrm{St}_{2} & |\cdot|^{\frac{1}{2}} \times|\cdot|^{\frac{3}{2}} & |\cdot|^{1} \mathrm{St}_{2} & |\cdot|^{\frac{3}{2}} \times|\cdot|^{\frac{3}{2}}
\end{array}\right),
\end{aligned}
$$

we have

$$
\left(m_{\underline{k}, \underline{k^{\prime}}}\right)_{\underline{k}, \underline{\underline{k}^{\prime}}}^{-1}=\left(\begin{array}{cccc}
1 & 0 & 0 & 0 \\
0 & 1 & 0 & 0 \\
0 & 1 & 1 & 0 \\
0 & 0 & 0 & 2
\end{array}\right)^{-1}=\left(\begin{array}{cccc}
1 & 0 & 0 & 0 \\
0 & 1 & 0 & 0 \\
0 & -1 & 1 & 0 \\
0 & 0 & 0 & \frac{1}{2}
\end{array}\right)
$$

Moreover

$$
\left(\begin{array}{c}
\Pi_{\underline{x}(2,0,0)}^{\epsilon} \\
\Pi_{\underline{x}(1,1,0)}^{\epsilon} \\
\Pi_{\underline{x}(0,2,0)}^{\epsilon} \\
\Pi_{\underline{x}(0,1,1)}^{\epsilon}
\end{array}\right)=\left(\begin{array}{c}
0 \\
\left.|\cdot|\right|^{\frac{1}{2}} \mathrm{St}_{3} \rtimes \mathbf{1}_{\mathrm{SO}_{1}(F)}+\pi_{\epsilon}(2,4)-\pi_{-\epsilon}(2,4) \\
|\cdot|{ }^{1} \mathrm{St}_{2} \rtimes \pi_{+}(2)+|\cdot|{ }^{\frac{1}{2}} \mathrm{St}_{3} \rtimes \mathbf{1}_{\mathrm{SO}_{1}(F)} \\
+\epsilon\left(|\cdot| \frac{1}{2} \rtimes \pi_{+}(4)+\pi_{+}(2,4)\right) \\
(1+\epsilon) \cdot \pi_{+}(2,2,2)
\end{array}\right) .
$$


Hence

$$
\begin{gathered}
\text { s.s.Jac } P_{2}\left(\pi_{\epsilon}(2,4,4)\right)=\left|\operatorname{det}_{2}\right|^{\frac{1}{2}} \otimes\left(|\cdot|^{\frac{1}{2}} \mathrm{St}_{3} \rtimes \mathbf{1}_{\mathrm{SO}_{1}(F)}+\pi_{\epsilon}(2,4)-\pi_{-\epsilon}(2,4)\right) \\
+|\cdot|^{\frac{1}{2}} \mathrm{St}_{2} \otimes\left(|\cdot|{ }^{1} \mathrm{St}_{2} \rtimes \pi_{+}(2)+|\cdot|^{\frac{1}{2}} \mathrm{St}_{3} \rtimes \mathbf{1}_{\mathrm{SO}_{1}(F)}\right. \\
\left.+\epsilon\left(|\cdot|^{\frac{1}{2}} \rtimes \pi_{+}(4)+\pi_{+}(2,4)\right)\right) \\
+\left(|\cdot|^{\frac{3}{2}} \times|\cdot|^{\frac{3}{2}}\right) \otimes \frac{1+\epsilon}{2} \cdot \pi_{+}(2,2,2) .
\end{gathered}
$$

(3) When $m=3$, we have $K_{\phi}^{(3)}=\{(2,1,0)>(1,2,0)>(1,1,1)>(0,3,0)>(0,2,1)\}$.

Since

$$
\begin{aligned}
& \left(\begin{array}{lllll}
\Delta_{\underline{x}(2,1,0)} & \Delta_{\underline{x}(1,2,0)} & \Delta_{\underline{x}(1,1,1)} & \Delta_{\underline{x}(0,3,0)} & \Delta_{\underline{x}(0,2,1)}
\end{array}\right) \\
& =\left(\mathrm{St}_{2} \times|\cdot|^{\frac{3}{2}}|\cdot|^{\frac{1}{2}} \times|\cdot|^{1} \mathrm{St}_{2}|\cdot|^{\frac{1}{2}} \times|\cdot|^{\frac{3}{2}} \times\left.|\cdot|^{\frac{3}{2}}|\cdot|\right|^{\frac{1}{2}} \mathrm{St}_{3}|\cdot|{ }^{1} \mathrm{St}_{2} \times|\cdot|^{\frac{3}{2}}\right),
\end{aligned}
$$

we have

$$
\left(m_{\underline{k}, \underline{k}^{\prime}}\right)_{\underline{k}, \underline{k^{\prime}}}^{-1}=\left(\begin{array}{ccccc}
1 & 0 & 0 & 0 & 0 \\
0 & 1 & 0 & 0 & 0 \\
0 & 0 & 2 & 0 & 0 \\
1 & 0 & 0 & 1 & 0 \\
0 & 0 & 2 & 0 & 1
\end{array}\right)^{-1}=\left(\begin{array}{ccccc}
1 & 0 & 0 & 0 & 0 \\
0 & 1 & 0 & 0 & 0 \\
0 & 0 & \frac{1}{2} & 0 & 0 \\
-1 & 0 & 0 & 1 & 0 \\
0 & 0 & -1 & 0 & 1
\end{array}\right) .
$$

\section{Moreover}

$$
\left(\begin{array}{c}
\Pi_{\frac{x}{\epsilon}(2,1,0)}^{\epsilon} \\
\Pi_{\frac{x}{\epsilon}(1,2,0)}^{\epsilon} \\
\Pi_{\underline{x}(1,1,1)}^{\epsilon} \\
\Pi_{\frac{x}{\epsilon}(0,3,0)}^{\epsilon} \\
\Pi_{\underline{x}(0,2,1)}^{\epsilon}
\end{array}\right)=\left(\begin{array}{c}
0 \\
|\cdot|{ }^{1} \mathrm{St}_{2} \rtimes \mathbf{1}_{\mathrm{SO}_{1}(F)}+\epsilon \cdot \pi_{+}(4) \\
2 \cdot \pi_{\epsilon}(2,2) \\
|\cdot| \frac{3}{2} \rtimes \pi_{+}(2)+\epsilon \cdot \pi_{+}(4) \\
(1+\epsilon)\left(\left.|\cdot|\right|^{\frac{1}{2}} \rtimes \pi_{+}(2)+\pi_{+}(2,2)\right)+\pi_{-}(2,2)
\end{array}\right)
$$

\section{Hence}

$$
\begin{aligned}
\text { s.s.Jac } P_{3}\left(\pi_{\epsilon}(2,4,4)\right) & =\left(\left.|\cdot|\right|^{\frac{1}{2}} \times|\cdot|{ }^{1} \mathrm{St}_{2}\right) \otimes\left(|\cdot|{ }^{1} \mathrm{St}_{2} \rtimes \mathbf{1}_{\mathrm{SO}_{1}(F)}+\epsilon \cdot \pi_{+}(4)\right) \\
& +\left(\left|\operatorname{det}_{2}\right|^{1} \times|\cdot| \frac{3}{2}\right) \otimes \pi_{\epsilon}(2,2) \\
& +|\cdot|{ }^{\frac{1}{2}} \mathrm{St}_{3} \otimes\left(|\cdot|^{\frac{3}{2}} \rtimes \pi_{+}(2)+\epsilon \cdot \pi_{+}(4)\right) \\
& +\left(|\cdot|{ }^{1} \mathrm{St}_{2} \times|\cdot|^{\frac{3}{2}}\right) \otimes\left((1+\epsilon)|\cdot|^{\frac{1}{2}} \rtimes \pi_{+}(2)+\pi_{+}(2,2)+\epsilon \cdot \pi_{-\epsilon}(2,2)\right) .
\end{aligned}
$$


(4) When $m=4$, we have $K_{\phi}^{(4)}=\{(2,2,0)>(2,1,1)>(1,3,0)>(1,2,1)>(0,4,0)>$ $(0,3,1)>(0,2,2)\}$. Since

$$
\left(\begin{array}{c}
\Delta_{\underline{x}}(2,2,0) \\
\Delta_{\underline{x}(2,1,1)} \\
\Delta_{\underline{x}(1,3,0)} \\
\Delta_{\underline{x}(1,2,1)}^{\underline{x}} \\
\Delta_{\underline{x}(0,4,0)}^{\underline{x}} \\
\Delta_{\underline{x}(0,3,1)}^{\underline{x}} \\
\Delta_{\underline{x}(0,2,2)}
\end{array}\right)=\left(\begin{array}{c}
\mathrm{St}_{2} \times|\cdot|{ }^{1} \mathrm{St}_{2} \\
\mathrm{St}_{2} \times\left.|\cdot|\right|^{\frac{3}{2}} \times\left.|\cdot|\right|^{\frac{3}{2}} \\
\left.|\cdot|\right|^{\frac{1}{2}} \times\left.|\cdot|\right|^{\frac{1}{2}} \mathrm{St}_{3} \\
|\cdot| \frac{1}{2} \times|\cdot|{ }^{1} \mathrm{St}_{2} \times|\cdot| \frac{3}{2} \\
\mathrm{St}_{4} \\
\left.|\cdot|\right|^{\frac{1}{2}} \mathrm{St}_{3} \times|\cdot|^{\frac{3}{2}} \\
|\cdot|{ }^{1} \mathrm{St}_{2} \times|\cdot|{ }^{1} \mathrm{St}_{2}
\end{array}\right)
$$

we have

$$
\left(m_{\underline{k}, \underline{k^{\prime}}}\right)_{\underline{k}, \underline{k^{\prime}}}^{-1}=\left(\begin{array}{ccccccc}
1 & 0 & 0 & 0 & 0 & 0 & 0 \\
0 & 2 & 0 & 0 & 0 & 0 & 0 \\
1 & 0 & 1 & 0 & 0 & 0 & 0 \\
0 & 0 & 0 & 1 & 0 & 0 & 0 \\
0 & 0 & 0 & 0 & 1 & 0 & 0 \\
0 & 2 & 0 & 0 & 0 & 1 & 0 \\
0 & 0 & 0 & 3 & 0 & 0 & 2
\end{array}\right)^{-1}=\left(\begin{array}{ccccccc}
1 & 0 & 0 & 0 & 0 & 0 & 0 \\
0 & \frac{1}{2} & 0 & 0 & 0 & 0 & 0 \\
-1 & 0 & 1 & 0 & 0 & 0 & 0 \\
0 & 0 & 0 & 1 & 0 & 0 & 0 \\
0 & 0 & 0 & 0 & 1 & 0 & 0 \\
0 & -1 & 0 & 0 & 0 & 1 & 0 \\
0 & 0 & 0 & -\frac{3}{2} & 0 & 0 & \frac{1}{2}
\end{array}\right) .
$$

Moreover

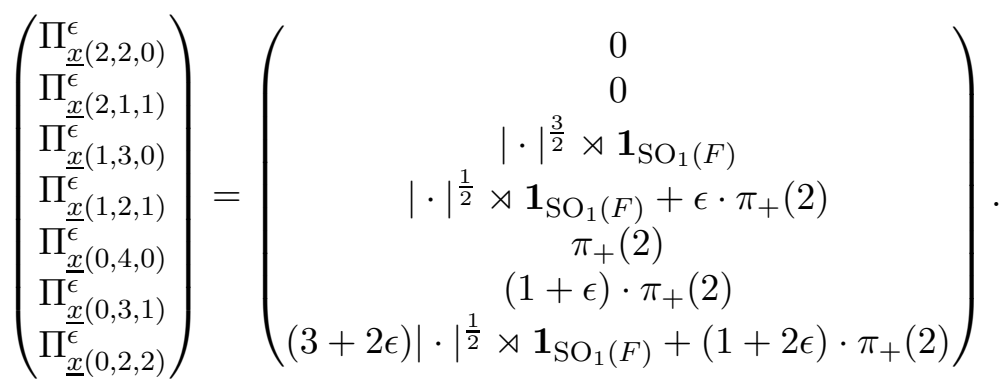

\section{Hence}

$$
\begin{aligned}
\operatorname{s.s.Jac}_{P_{4}}\left(\pi_{\epsilon}(2,4,4)\right) & =\left.\left(\left.|\cdot|\right|^{\frac{1}{2}} \times|\cdot|{ }^{\frac{1}{2}} \mathrm{St}_{3}\right) \otimes|\cdot|\right|^{\frac{3}{2}} \rtimes \mathbf{1}_{\mathrm{SO}_{1}(F)} \\
& +\left(|\cdot|{ }^{1} \mathrm{St}_{2} \times\left|\operatorname{det}_{2}\right|^{1}\right) \otimes\left(\left.|\cdot|\right|^{\frac{1}{2}} \rtimes \mathbf{1}_{\mathrm{SO}_{1}(F)}+\epsilon \cdot \pi_{+}(2)\right) \\
& +\mathrm{St}_{4} \otimes \pi_{+}(2) \\
& +\left(|\cdot|{ }^{\frac{1}{2}} \mathrm{St}_{3} \times|\cdot|^{\frac{3}{2}}\right) \otimes(1+\epsilon) \cdot \pi_{+}(2) \\
& +\left(|\cdot|{ }^{1} \mathrm{St}_{2} \times|\cdot|{ }^{1} \mathrm{St}_{2}\right) \otimes\left((1+\epsilon)|\cdot| \frac{1}{2} \rtimes \mathbf{1}_{\mathrm{SO}_{1}(F)}+\frac{1+\epsilon}{2} \cdot \pi_{+}(2)\right) .
\end{aligned}
$$


(5) When $m=5$, we have $K_{\phi}^{(5)}=\{(2,3,0)>(2,2,1)>(1,4,0)>(1,3,1)>(1,2,2)>$ $(0,4,1)>(0,3,2)\}$. Since

$$
\left(\begin{array}{c}
\Delta_{\underline{x}(2,3,0)} \\
\Delta_{\underline{x}(2,2,1)} \\
\Delta_{\underline{x}(1,4,0)} \\
\Delta_{\underline{x}(1,3,1)}^{\underline{x}(1,2)} \\
\Delta_{\underline{x}(1,2)}^{\Delta_{x}(0,4,1)} \\
\Delta_{\underline{x}(0,3,2)}
\end{array}\right)=\left(\begin{array}{c}
\mathrm{St}_{2} \times|\cdot|^{\frac{1}{2}} \mathrm{St}_{3} \\
\mathrm{St}_{2} \times|\cdot|^{1} \mathrm{St}_{2} \times|\cdot|^{\frac{3}{2}} \\
|\cdot|^{\frac{1}{2}} \times \mathrm{St}_{4} \\
\left.|\cdot|\right|^{\frac{1}{2}} \times\left.|\cdot|\right|^{\frac{1}{2}} \mathrm{St}_{3} \times|\cdot|^{\frac{3}{2}} \\
\left.|\cdot|\right|^{\frac{1}{2}} \times|\cdot|{ }^{1} \mathrm{St}_{2} \times|\cdot|{ }^{1} \mathrm{St}_{2} \\
\mathrm{St}_{4} \times\left.|\cdot| \cdot\right|^{\frac{3}{2}} \\
|\cdot|^{\frac{1}{2}} \mathrm{St}_{3} \times|\cdot|{ }^{1} \mathrm{St}_{2}
\end{array}\right)
$$

we have

$$
\left(m_{\underline{k}, \underline{k}^{\prime}}\right)_{\underline{k}, \underline{k^{\prime}}}^{-1}=\left(\begin{array}{ccccccc}
1 & 0 & 0 & 0 & 0 & 0 & 0 \\
0 & 1 & 0 & 0 & 0 & 0 & 0 \\
0 & 0 & 1 & 0 & 0 & 0 & 0 \\
0 & 1 & 0 & 1 & 0 & 0 & 0 \\
0 & 0 & 0 & 0 & 2 & 0 & 0 \\
0 & 0 & 0 & 0 & 0 & 1 & 0 \\
0 & 2 & 0 & 1 & 0 & 0 & 1
\end{array}\right)^{-1}=\left(\begin{array}{ccccccc}
1 & 0 & 0 & 0 & 0 & 0 & 0 \\
0 & 1 & 0 & 0 & 0 & 0 & 0 \\
0 & 0 & 1 & 0 & 0 & 0 & 0 \\
0 & -1 & 0 & 1 & 0 & 0 & 0 \\
0 & 0 & 0 & 0 & \frac{1}{2} & 0 & 0 \\
0 & 0 & 0 & 0 & 0 & 1 & 0 \\
0 & -1 & 0 & -1 & 0 & 0 & 1
\end{array}\right) .
$$

Moreover

Hence

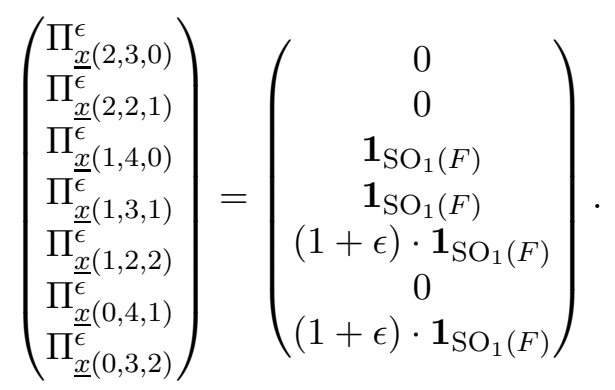

$$
\begin{aligned}
\operatorname{s.S.Jac} P_{5}\left(\pi_{\epsilon}(2,4,4)\right) & =\left(|\cdot|^{\frac{1}{2}} \times \mathrm{St}_{4}\right) \otimes \mathbf{1}_{\mathrm{SO}_{1}(F)} \\
& +\left(|\cdot|^{\frac{1}{2}} \mathrm{St}_{3} \times\left|\operatorname{det}_{2}\right|^{1}\right) \otimes \mathbf{1}_{\mathrm{SO}_{1}(F)} \\
& +\left(|\cdot|^{\frac{1}{2}} \times|\cdot|{ }^{1} \mathrm{St}_{2} \times|\cdot|{ }^{1} \mathrm{St}_{2}\right) \otimes \frac{1+\epsilon}{2} \cdot \mathbf{1}_{\mathrm{SO}_{1}(F)} \\
& +\left(|\cdot|^{\frac{1}{2}} \mathrm{St}_{3} \times|\cdot|{ }^{1} \mathrm{St}_{2}\right) \otimes(1+\epsilon) \cdot \mathbf{1}_{\mathrm{SO}_{1}(F)} .
\end{aligned}
$$

Remark 4.7. In Theorem 4.3, one can replace $\Delta_{\underline{x}(\underline{k})}$ with its unique irreducible subrepresentation $\tau_{\underline{x}(\underline{k})}$. Then one should consider the matrix $\left(M_{\underline{k}, \underline{l}}\right)=\left(\operatorname{dim}_{\mathbb{C}} \operatorname{Jac}_{\rho|\cdot| \underline{x}(\underline{k})}\left(\tau_{\underline{x}(\underline{l})}\right)\right)$. One might seem that $\left(M_{\underline{k}, \underline{l}}\right)$ easier than $\left(m_{\underline{k}, \underline{l}}\right)$. For instance, if $\phi$ is in Example 4.5, all $\left(M_{\underline{k}, \underline{l}}\right)$ are the identity matrix, but not so is some $\left(m_{\underline{k}, \underline{l}}\right)$. However, in general, $\left(M_{\underline{k}, \underline{l}}\right)$ is not always diagonal. In Example 4.6 , such a non-diagonal $\left(M_{\underline{k}, \underline{l}}\right)$ appears.

\section{Proof of Main theorems}

In this section, we prove main theorems (Theorems 4.2 and 4.3 ). 
5.1. The case of higher multiplicity. We prove Theorem 4.2 (1) in this subsection. It immediately follows from the case of $\phi \in \Phi_{\text {disc }}(G)$ together with Tadic's formula (Corollary [2.8).

Proof of Theorem 4.2 (1). We prove the assertion by induction on $m$. Since

$$
\pi(\phi, \eta)=\operatorname{St}(\rho, 2 x+1) \rtimes \pi\left(\phi-\left(\rho \otimes S_{2 x+1}\right)^{\oplus 2}, \eta\right),
$$

by Corollary 2.8, we have

$$
\begin{aligned}
\operatorname{Jac}_{\rho|\cdot| x}(\pi(\phi, \eta)) & =2 \cdot\langle\rho ; x, \ldots,-(x-1)\rangle \rtimes \pi\left(\phi-\left(\rho \otimes S_{2 x+1}\right)^{\oplus 2}, \eta\right) \\
& +\operatorname{St}(\rho, 2 x+1) \rtimes \operatorname{Jac}_{\rho|\cdot| x}\left(\pi\left(\phi-\left(\rho \otimes S_{2 x+1}\right)^{\oplus 2}, \eta\right)\right) .
\end{aligned}
$$

This proves the assertion when $m=3$ or $m=4$. When $m \geq 5$, since

$$
\begin{aligned}
& \operatorname{St}(\rho, 2 x+1) \times\langle\rho ; x, \ldots,-(x-1)\rangle \rtimes \pi\left(\phi-\left(\rho \otimes S_{2 x+1}\right)^{\oplus 4}, \eta\right) \\
& \cong\langle\rho ; x, \ldots,-(x-1)\rangle \times \operatorname{St}(\rho, 2 x+1) \rtimes \pi\left(\phi_{0} \oplus\left(\phi-\left(\rho \otimes S_{2 x+1}\right)^{\oplus 4}\right), \eta\right) \\
& \cong\langle\rho ; x, \ldots,-(x-1)\rangle \rtimes \pi\left(\phi-\left(\rho \otimes S_{2 x+1}\right)^{\oplus 2}, \eta\right),
\end{aligned}
$$

we obtain the assertion by the induction hypothesis.

5.2. The case of multiplicity one. Next, we prove Theorem $4.2(2)$. Let $\phi=\phi_{0} \oplus\left(\rho \bigotimes S_{2 x+1}\right)$ with $\rho \otimes S_{2 x+1} \not \subset \phi_{0}$, and $\eta \in \widehat{\mathcal{A}_{\phi}}$. Set

$$
\phi^{\prime}=\phi-\left(\rho \otimes S_{2 x+1}\right) \oplus\left(\rho \otimes S_{2 x-1}\right) .
$$

Proof of Theorem 4.2 (2). First, we assume that $x>0$ and $\pi\left(\phi^{\prime}, \eta^{\prime}\right) \neq 0$. We apply Møglin's construction to $\Pi_{\phi^{\prime}}$. Write

$$
\phi^{\prime}=\left(\bigoplus_{i=1}^{t} \rho \otimes S_{a_{i}}\right) \oplus \phi_{e}^{\prime}
$$

with $a_{1} \leq \cdots \leq a_{t}$ and $\rho \otimes S_{a} \not \subset \phi_{e}^{\prime}$ for any $a>0$. Set

$$
t_{0}=\max \left\{i \in\{1, \ldots, t\} \mid t_{i}=2 x-1\right\} .
$$

Take a new $L$-parameter

such that

$$
\phi_{\gg}^{\prime}=\left(\bigoplus_{i=1}^{t} \rho \otimes S_{a_{i}^{\prime}}\right) \oplus \phi_{e}^{\prime}
$$

- $a_{1}^{\prime}<\cdots<a_{t}^{\prime}$

- $a_{i}^{\prime} \geq a_{i}$ and $a_{i}^{\prime} \equiv a_{i} \bmod 2$ for any $i$

- $a_{t_{0}}^{\prime} \geq 2 x+1$.

We can identify $\mathcal{A}_{\phi_{\gg}^{\prime}}$ with $\mathcal{A}_{\phi^{\prime}}$ canonically. Let $\eta_{\gg}^{\prime} \in \widehat{\mathcal{A}_{\phi_{\gg}^{\prime}}}$ be the character corresponding to $\eta^{\prime} \in \widehat{\mathcal{A}_{\phi^{\prime}}}$. Then Theorem 3.3 says that

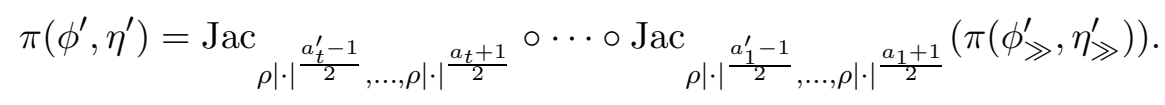

When $i=t_{0}$, we note that

$$
\mathrm{Jac} \underset{\rho|\cdot| \frac{a_{t_{0}}^{\prime}-1}{2}, \ldots, \rho|\cdot|{\frac{a}{t_{0}+1}}_{2}}{2}=\mathrm{Jac}_{\rho|\cdot| x} \circ \mathrm{Jac} \underset{\rho|\cdot| \frac{a_{t_{0}}^{\prime}-1}{2}, \ldots, \rho|\cdot| \frac{a_{t_{0}}+3}{2}}{ } .
$$


Since $\phi^{\prime}$ does not contain $\rho \bigotimes S_{2 x+1}$, for $i>t_{0}$ and $a_{i}<2 x^{\prime}+1 \leq a_{i}^{\prime}$ with $2 x^{\prime}+1 \equiv a_{i} \bmod 2$, we have $x^{\prime}-x>1$. By Lemma 2.5 (2), we see that $\pi\left(\phi^{\prime}, \eta^{\prime}\right)$ is the image of

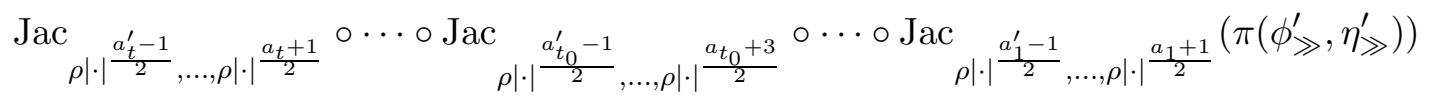

under $\operatorname{Jac}_{\rho|\cdot| x}$. However, by applying Theorem 3.3 again, we see that this representation is isomorphic to $\pi(\phi, \eta)$. Therefore $\pi\left(\phi^{\prime}, \eta^{\prime}\right)=\operatorname{Jac}_{\rho|\cdot| x}(\pi(\phi, \eta))$, as desired.

Next, we assume that $\pi\left(\phi^{\prime}, \eta^{\prime}\right)=0$. We claim that $\operatorname{Jac}_{\rho|\cdot| x}(\pi(\phi, \eta))=0$. When $\phi \in \Phi_{\text {disc }}(G)$, this was proven in [X17a, Lemma 7.3]. When $\phi \in \Phi_{\mathrm{gp}}(G) \backslash \Phi_{\mathrm{disc}}(G)$, there exists an irreducible representation $\phi_{1}$ which is contained in $\phi$ with multiplicity at least two. Then $\pi(\phi, \eta)$ is a subrepresentation of $\tau_{1} \rtimes \pi\left(\phi-\phi_{1}^{\oplus 2}, \eta\right)$, where $\tau_{1}$ is the irreducible tempered representation of $\mathrm{GL}_{k}(F)$ corresponding to $\phi_{1}$. Since $\rho \otimes S_{2 x+1}$ is contained in $\phi$ with multiplicity one, we have $\phi_{1} \not \rho \otimes S_{2 x+1}$. This implies that

$$
\operatorname{Jac}_{\rho|\cdot| x}\left(\tau_{1} \rtimes \pi\left(\phi-\phi_{1}^{\oplus 2}, \eta\right)\right)=\tau_{1} \rtimes \operatorname{Jac}_{\rho|\cdot| x}\left(\pi\left(\phi-\phi_{1}^{\oplus 2}, \eta\right)\right) .
$$

By the induction hypothesis, $\operatorname{Jac}_{\rho|\cdot| x}\left(\pi\left(\phi-\phi_{1}^{\oplus 2}, \eta\right)\right)=0$ unless $\phi_{1}=\rho \otimes S_{2 x-1}$ and $\phi$ contains it with multiplicity exactly two. In this case, one can take $\eta_{-} \in \widehat{\mathcal{A}_{\phi}}$ such that $\pi\left(\phi, \eta_{-}\right) \neq 0$ and

$$
\pi(\phi, \eta) \oplus \pi\left(\phi, \eta_{-}\right)=\mathrm{St}(\rho, 2 x-1) \rtimes \pi\left(\phi-\phi_{1}^{\oplus 2}, \eta\right) .
$$

Then by the first case, we see that $\operatorname{Jac}_{\rho|\cdot| x}\left(\pi\left(\phi, \eta_{-}\right)\right) \neq 0$ and $\operatorname{St}(\rho, 2 x-1) \rtimes \operatorname{Jac}_{\rho|\cdot| x}(\pi(\phi-$ $\left.\left.\phi_{1}^{\oplus 2}, \eta\right)\right)$ is irreducible. Hence $\operatorname{Jac}_{\rho|\cdot| x}(\pi(\phi, \eta))$ must be zero. This completes the proof of Theorem 4.2 (2).

By the same argument as the last part, one can prove that $\operatorname{Jac}_{\rho}(\pi(\phi, \eta))=0$ when $x=0$ and $m=1$.

5.3. Description of small standard modules. Before proving Theorem 4.2 (3), we describe the structures of some standard modules.

Lemma 5.1. Let $\phi \in \Phi_{\text {disc }}(G)$. Suppose that $x>0$, and $\phi \supset \rho \otimes S_{2 x-1}$ but $\phi \not \supset \rho \otimes S_{2 x+1}$. Let $\eta \in \widehat{\mathcal{A}_{\phi}}$ such that $\pi(\phi, \eta) \neq 0$. We set

- $\Pi=\rho|\cdot|^{x} \rtimes \pi(\phi, \eta)$ to be a standard module;

- $\sigma$ to be the unique irreducible quotient of $\Pi$;

- $\phi^{\prime}=\phi-\left(\rho \otimes S_{2 x-1}\right) \oplus\left(\rho \otimes S_{2 x+1}\right)$;

- $\eta^{\prime} \in \widehat{\mathcal{A}_{\phi^{\prime}}}$ to be the character corresponding to $\eta \in \widehat{\mathcal{A}_{\phi}}$ via the canonical identification $\mathcal{A}_{\phi^{\prime}}=\mathcal{A}_{\phi}$.

Then there exists an exact sequence

$$
0 \longrightarrow \pi\left(\phi^{\prime}, \eta^{\prime}\right) \longrightarrow \Pi \longrightarrow \sigma \longrightarrow 0
$$

In particular, П has length two.

Proof. We note that $\pi\left(\phi^{\prime}, \eta^{\prime}\right)$ is an irreducible subrepresentation of $\Pi$ by Theorem 4.2 (2) and Lemma 2.5 (1).

If $\sigma^{\prime}$ is an irreducible subquotient of $\Pi$ which is non-tempered, by Tadić's formula and Casselman's criterion, there exists a maximal parabolic subgroup $P_{k}$ of $G(F)$ such that 
s.s. $\operatorname{Jac}_{P_{k}}\left(\sigma^{\prime}\right)$ contains an irreducible representation of the form $\left(\rho|\cdot|^{-x} \times \tau\right) \otimes \sigma_{0}$. In particular, we have $\operatorname{Jac}_{\rho|\cdot|^{-x}}\left(\sigma^{\prime}\right) \neq 0$. However, since $\operatorname{Jac}_{\rho|\cdot|-x}(\Pi)=\pi(\phi, \eta)$ is irreducible, we see that $\sigma^{\prime}=\sigma$, i.e., $\Pi$ has only one irreducible non-tempered subquotient.

Let $\Pi^{\text {sub }}$ be the maximal proper subrepresentation of $\Pi$, i.e., $\Pi / \Pi^{\text {sub }} \cong \sigma$. By the above argument, all irreducible subquotients of $\Pi^{\text {sub }}$ must be tempered. Since they have the same cuspidal support, they share the same Plancherel measure. This implies that all irreducible subquotients of $\Pi^{\text {sub }}$ belong to the same $L$-packet $\Pi_{\phi^{\prime}}$ (see [GI16, Lemma A.6]), so that they are all discrete series. Hence $\Pi^{\text {sub }}$ is semisimple. In particular, any irreducible subquotient $\pi^{\prime}$ of $\Pi^{\mathrm{sub}}$ is a subrepresentation of $\Pi$, so that $\mathrm{Jac}_{\rho|\cdot| x}\left(\pi^{\prime}\right) \neq 0$. However, since $\mathrm{Jac}_{\rho|\cdot| x}(\Pi)=$ $\pi(\phi, \eta)$ is irreducible, $\Pi$ has only one irreducible subrepresentation. Therefore $\Pi^{\text {sub }}=\pi\left(\phi^{\prime}, \eta^{\prime}\right)$. This completes the proof.

We describe the standard module appearing in Theorem 4.2 (3). When $x=1 / 2$, the standard module was described in Lemma 5.1. Hence we assume $x>1 / 2$.

Proposition 5.2. Let $\phi \in \Phi_{\mathrm{gp}}(G)$. Suppose that $x>1 / 2$ and $\phi \not \supset \rho \otimes S_{2 x+1}$. Let $\eta \in \widehat{\mathcal{A}_{\phi}}$ such that $\pi(\phi, \eta) \neq 0$. We set

- $\Pi=\langle\rho ; x, x-1, \ldots,-(x-1)\rangle \rtimes \pi(\phi, \eta)$ to be a standard module;

- $\sigma$ to be the unique irreducible quotient of $\Pi$;

- $\phi^{\prime}=\phi \oplus\left(\rho \otimes S_{2 x-1}\right) \oplus\left(\rho \otimes S_{2 x+1}\right)$;

- $\eta_{+}^{\prime}$ and $\eta_{-}^{\prime}$ to be the two distinct characters of $\widehat{\mathcal{A}_{\phi^{\prime}}}$ such that $\eta_{ \pm}^{\prime} \mid \mathcal{A}_{\phi}=\eta$ and $\eta_{ \pm}^{\prime}\left(z_{\phi^{\prime}}\right)=$ 1 .

Then there exists an exact sequence

$$
0 \longrightarrow \pi\left(\phi^{\prime}, \eta_{+}^{\prime}\right) \oplus \pi\left(\phi^{\prime}, \eta_{-}^{\prime}\right) \longrightarrow \Pi \longrightarrow \sigma \longrightarrow 0 .
$$

In particular, $\Pi$ has length 2 or 3 according to $\phi \supset \rho \bigotimes S_{2 x-1}$ or not.

Proof. First, we show that there is an inclusion $\pi\left(\phi^{\prime}, \eta_{\epsilon}^{\prime}\right) \hookrightarrow \Pi$ for each $\epsilon \in\{ \pm 1\}$. To do this, we may assume that $\pi\left(\phi^{\prime}, \eta_{\epsilon}^{\prime}\right) \neq 0$. Note that $\phi^{\prime}$ contains $\rho \otimes S_{2 x+1}$ with multiplicity one. By Theorem $4.2(2)$, we see that $\operatorname{Jac}_{\rho|\cdot| x}\left(\pi\left(\phi^{\prime}, \eta_{\epsilon}^{\prime}\right)\right)$ is nonzero and is an irreducible subrepresentation of $\operatorname{St}(\rho, 2 x-1) \rtimes \pi(\phi, \eta)$. By Lemma 2.5 (1), we have an inclusion

$$
\pi\left(\phi^{\prime}, \eta_{\epsilon}^{\prime}\right) \hookrightarrow \rho|\cdot|^{x} \times \operatorname{St}(\rho, 2 x-1) \rtimes \pi(\phi, \eta) .
$$

Since $\Pi$ is a subrepresentation of $\rho|\cdot|^{x} \times \operatorname{St}(\rho, 2 x-1) \rtimes \pi(\phi, \eta)$ such that

$$
\operatorname{Jac}_{\rho|\cdot|^{x}}\left(\rho|\cdot|^{x} \times \operatorname{St}(\rho, 2 x-1) \rtimes \pi(\phi, \eta)\right)=\operatorname{Jac}_{\rho|\cdot|^{x}}(\Pi),
$$

the above inclusion factors through $\pi\left(\phi^{\prime}, \eta_{\epsilon}^{\prime}\right) \hookrightarrow \Pi$.

If s.s. $\operatorname{Jac}_{P_{k}}(\Pi)$ contains an irreducible representation $\tau \otimes \pi_{0}$ such that the central character of $\tau$ is of the form $\chi|\cdot|^{s}$ with $\chi$ unitary and $s<0$, by Tadić's formula (Theorem 2.7) and Casselman's criterion, $\tau=|\cdot|^{-\frac{1}{2}} \operatorname{St}(\rho, 2 x) \times\left(\times_{i=1}^{r} \tau_{i}\right)$, where $\tau_{i}$ is a discrete series representation of $\mathrm{GL}_{k_{i}}(F)$ such that the corresponding irreducible representation $\phi_{i}$ of $W_{F} \times \mathrm{SL}_{2}(\mathbb{C})$ is contained in $\phi$ with multiplicity at least two, and $\pi_{0}=\pi\left(\phi_{0}, \eta_{0}\right)$ with $\phi_{0}=\phi-\left(\oplus_{i=1}^{r} \phi_{i}\right)^{\oplus 2}$ and $\eta_{0}=\eta \mid \mathcal{A}_{\phi_{0}}$. Since such an irreducible representation $\tau \otimes \pi_{0}$ is also contained in $\operatorname{s.s.Jac}_{P_{k}}(\sigma)$, we see that $\sigma$ is the unique irreducible non-tempered subquotient of $\Pi$. Namely, if we let $\Pi^{\text {sub }}$ be the maximal proper subrepresentation of $\Pi$, i.e., $\Pi / \Pi^{\text {sub }} \cong \sigma$, then all irreducible subquotients of $\Pi^{\text {sub }}$ must be tempered. Moreover since these irreducible subquotients have 
the same cuspidal support so that they share the same Plancherel measure, they belong to the same $L$-packet $\Pi_{\phi^{\prime}}$ (see [GI16, Lemma A.6]).

Now we show that $\Pi^{\text {sub }}$ is isomorphic to $\pi\left(\phi^{\prime}, \eta_{+}^{\prime}\right) \oplus \pi\left(\phi^{\prime}, \eta_{-}^{\prime}\right)$. We separate the cases as follows:

- $\phi$ is discrete and $\rho \otimes S_{2 x-1} \not \subset \phi$;

- $\phi$ is discrete and $\rho \bigotimes S_{2 x-1} \subset \phi$;

- $\phi$ is general.

When $\phi$ is discrete and $\rho \otimes S_{2 x-1} \not \subset \phi$, we note that $\phi^{\prime}=\phi \oplus\left(\rho \otimes S_{2 x-1}\right) \oplus\left(\rho \otimes S_{2 x+1}\right)$ is also discrete. Then since all irreducible subquotients of $\Pi^{\text {sub }}$ are discrete series, $\Pi^{\text {sub }}$ is semisimple. In particular, any irreducible subquotient $\pi^{\prime}$ of $\Pi^{\text {sub }}$ is a subrepresentation of $\Pi$ so that $\operatorname{Jac}_{\rho|\cdot| x}\left(\pi^{\prime}\right) \neq 0$. However, since $\operatorname{Jac}_{\rho|\cdot| x}(\Pi)=\operatorname{Jac}_{\rho|\cdot| x}\left(\pi\left(\phi^{\prime}, \eta_{+}^{\prime}\right) \oplus \pi\left(\phi^{\prime}, \eta_{-}^{\prime}\right)\right)$, we have $\Pi^{\mathrm{sub}} \cong \pi\left(\phi^{\prime}, \eta_{+}^{\prime}\right) \oplus \pi\left(\phi^{\prime}, \eta_{-}^{\prime}\right)$.

When $\phi$ is discrete and $\rho \otimes S_{2 x-1} \subset \phi$, any irreducible subquotient $\pi^{\prime}$ of $\Pi^{\text {sub }}$ belongs to $\Pi_{\phi^{\prime}}$ with $\phi^{\prime}=\phi_{0}^{\prime} \oplus\left(\rho \otimes S_{2 x-1}\right)^{\oplus 2}$, where $\phi_{0}^{\prime}=\phi-\left(\rho \otimes S_{2 x-1}\right) \oplus\left(\rho \otimes S_{2 x+1}\right)$ is discrete such that $\rho \otimes S_{2 x-1} \not \subset \phi_{0}^{\prime}$. Hence the Jacquet module s.s. $\operatorname{Jac}_{P_{d(2 x-1)}}\left(\pi^{\prime}\right)$ contains an irreducible representation of the form $\operatorname{St}(\rho, 2 x-1) \otimes \pi_{0}^{\prime}$. By Tadić's formula (Corollary 2.8), the sum of irreducible representations of this form appearing in $\operatorname{s.s.Jac}_{P_{d(2 x-1)}}(\Pi)$ is

$$
\operatorname{St}(\rho, 2 x-1) \otimes \operatorname{s.s.}\left(\rho|\cdot|^{x} \rtimes \pi(\phi, \eta)\right) .
$$

By Lemma 5.1, we have an exact sequence

$$
0 \longrightarrow \pi\left(\phi_{0}^{\prime}, \eta_{0}^{\prime}\right) \longrightarrow \rho|\cdot|^{x} \rtimes \pi(\phi, \eta) \longrightarrow \sigma^{\prime} \longrightarrow 0,
$$

where $\sigma^{\prime}$ is the unique irreducible quotient of $\left.\rho|\cdot|\right|^{x} \rtimes \pi(\phi, \eta)$, and $\eta_{0}^{\prime} \in \widehat{\mathcal{A}_{\phi_{0}^{\prime}}}$ is the character corresponding to $\eta \in \widehat{\mathcal{A}_{\phi}}$. Now there exists $\epsilon \in\{ \pm 1\}$ such that $\pi\left(\phi^{\prime}, \eta_{-\epsilon}^{\prime}\right)=0$. Moreover, s.s. $\operatorname{Jac}_{P_{d(2 x-1)}}\left(\pi\left(\phi^{\prime}, \eta_{\epsilon}^{\prime}\right)\right) \supset \operatorname{St}(\rho, 2 x-1) \otimes \pi\left(\phi_{0}^{\prime}, \eta_{0}^{\prime}\right)$ since

$$
\eta_{\epsilon}^{\prime}\left(\alpha_{\rho \bigotimes S_{2 x+1}}\right)=\eta_{\epsilon}^{\prime}\left(\alpha_{\rho \bigotimes S_{2 x-1}}\right)=\eta\left(\alpha_{\rho \bigotimes S_{2 x-1}}\right)=\eta_{0}\left(\alpha_{\rho \bigotimes S_{2 x-1}}\right) .
$$

On the other hand, since $\sigma \hookrightarrow \operatorname{St}(\rho, 2 x-1) \times \rho|\cdot|^{-x} \rtimes \pi(\phi, \eta)$, we see that $\operatorname{Jac}_{P_{d(2 x-1)}}(\sigma)$ is nonzero and contains $\operatorname{St}(\rho, 2 x-1) \otimes \sigma^{\prime}$. Hence

$$
\text { s.s. } \operatorname{Jac}_{P_{d(2 x-1)}}(\Pi)-\text { s.s.Jac } P_{d(2 x-1)}\left(\pi\left(\phi^{\prime}, \eta_{\epsilon}^{\prime}\right)\right)-\text { s.s.Jac } \operatorname{Jac}_{P_{d(2 x-1)}}(\sigma)
$$

has no irreducible representation of the form $\operatorname{St}(\rho, 2 x-1) \otimes \pi_{0}^{\prime}$. This shows that $\Pi^{\mathrm{sub}}=$ $\pi\left(\phi^{\prime}, \eta_{\epsilon}^{\prime}\right)$.

In general, we prove the claim by induction on the dimension of $\phi$. When $\phi$ is not discrete, there exists an irreducible representation $\phi_{1}$ of $W_{F} \times \mathrm{SL}_{2}(\mathbb{C})$ which $\phi$ contains with multiplicity at least two. Note that $\phi_{1} \approx \rho \otimes S_{2 x+1}$. Set $\phi_{0}=\phi-\phi_{1}^{\oplus 2}$, and $\eta_{0}=\eta \mid \mathcal{A}_{\phi_{0}}$. Take $\Pi_{0}, \sigma_{0}, \phi_{0}^{\prime}$ and $\eta_{0, \epsilon}^{\prime} \in \widehat{\mathcal{A}_{\phi_{0}^{\prime}}}$ as in the statement of the proposition. By induction hypothesis, we have an exact sequence

$$
0 \longrightarrow \pi\left(\phi_{0}^{\prime}, \eta_{0,+}^{\prime}\right) \oplus \pi\left(\phi_{0}^{\prime}, \eta_{0,-}^{\prime}\right) \longrightarrow \Pi_{0} \longrightarrow \sigma_{0} \longrightarrow 0
$$

Let $\tau$ be the irreducible discrete series representation of $\mathrm{GL}_{k}(F)$ corresponding to $\phi_{1}$. The above exact sequence remains exact after taking the parabolic induction functor $\pi_{0} \mapsto \tau \rtimes \pi_{0}$. Note that $\tau \times\langle\rho ; x, x-1, \ldots,-(x-1)\rangle \cong\langle\rho ; x, x-1, \ldots,-(x-1)\rangle \times \tau$ by Theorem 2.1. Since $\sigma_{0}$ is unitary, the parabolic induction $\tau \rtimes \sigma_{0}$ is semisimple. In particular, any irreducible subquotient of $\tau \rtimes \sigma_{0}$ is non-tempered. Considering the cases where 
- $\phi$ contains $\phi_{1}$ with multiplicity more than two;

- $\phi$ contains $\phi_{1}$ with multiplicity exactly two and $\phi_{1} \neq \rho \otimes S_{2 x-1}$;

- $\phi$ contains $\phi_{1}$ with multiplicity exactly two and $\phi_{1} \cong \rho \otimes S_{2 x-1}$

separately, we see that $\Pi^{\text {sub }} \cong \pi\left(\phi^{\prime}, \eta_{+}^{\prime}\right) \oplus \pi\left(\phi^{\prime}, \eta_{-}^{\prime}\right)$ in all cases. This completes the proof.

5.4. The case of multiplicity two. Finally, we prove Theorem 4.2 (3).

Lemma 5.3. Let $\phi \in \Phi_{\mathrm{gp}}(G), \eta \in \widehat{\mathcal{A}_{\phi}}$ and $x>0$. Suppose that $\phi$ contains both $\rho \otimes S_{2 x+1}$ and $\rho \otimes S_{2 x+3}$ with multiplicity one. Then we have

$$
\operatorname{Jac}_{\left.\rho|\cdot|\right|^{x+1}, \rho\left|\cdot{ }^{x}, \rho\right| \cdot \mid x}(\pi(\phi, \eta)) \subset 2 \cdot \operatorname{Jac}_{\rho|\cdot|^{x}, \rho|\cdot|^{x+1}, \rho|\cdot|^{x}}(\pi(\phi, \eta)) .
$$

Proof. We may assume that $\operatorname{Jac}_{\rho|\cdot|{ }^{x+1}, \rho|\cdot|^{x}, \rho|\cdot| x}(\pi(\phi, \eta)) \neq 0$. By Lemma 2.5 (1), there exists an irreducible subquotient $\sigma$ of this Jacquet module such that

$$
\pi(\phi, \eta) \hookrightarrow \rho|\cdot|{ }^{x+1} \times \rho|\cdot|^{x} \times \rho|\cdot|^{x} \rtimes \sigma .
$$

Since there exists an exact sequence

$$
0 \longrightarrow\langle\rho ; x+1, x\rangle \longrightarrow \rho|\cdot|^{x+1} \times \rho|\cdot|^{x} \longrightarrow\langle\rho ; x, x+1\rangle \longrightarrow 0,
$$

where $\langle\rho ; x, x+1\rangle$ is the unique irreducible subrepresentation of $\rho|\cdot|{ }^{x} \times \rho|\cdot|{ }^{x+1}$, we see that $\pi(\phi, \eta)$ is a subrepresentation of $\langle\rho ; x+1, x\rangle \times \rho|\cdot|{ }^{x} \rtimes \sigma$ or $\langle\rho ; x, x+1\rangle \times \rho|\cdot|{ }^{x} \rtimes \sigma$. Since $\langle\rho ; x+1, x\rangle \times \rho|\cdot|^{x} \cong \rho|\cdot|^{x} \times\langle\rho ; x+1, x\rangle$ and $\langle\rho ; x, x+1\rangle \times \rho|\cdot|^{x} \cong \rho|\cdot|{ }^{x} \times\langle\rho ; x, x+1\rangle$, we have $\operatorname{Jac}_{\rho|\cdot| x}(\pi(\phi, \eta)) \neq 0$.

By Theorem $4.2(2), \operatorname{Jac}_{\rho|\cdot| x+1}(\pi(\phi, \eta)) \neq 0$ and $\operatorname{Jac}_{\rho|\cdot| x}(\pi(\phi, \eta)) \neq 0$ imply that $\sigma^{\prime}=$ $\operatorname{Jac}_{\rho|\cdot|{ }^{x}, \rho|\cdot|{ }^{x+1}, \rho \mid \cdot{ }^{x}}(\pi(\phi, \eta))$ is nonzero and irreducible. Moreover, we have $\operatorname{Jac}_{\rho|\cdot| x}\left(\sigma^{\prime}\right)=0$ and $\operatorname{Jac}_{\rho|\cdot| x+1}\left(\sigma^{\prime}\right)=0$. By Lemma 2.5 (1), we have an inclusion

$$
\pi(\phi, \eta) \hookrightarrow \rho|\cdot|^{x} \times \rho|\cdot|{ }^{x+1} \times \rho|\cdot|^{x} \rtimes \sigma^{\prime} .
$$

Since

$$
\operatorname{Jac}_{\rho|\cdot|{ }^{x+1}, \rho|\cdot| x, \rho|\cdot| x}\left(\rho|\cdot|{ }^{x} \times \rho|\cdot|{ }^{x+1} \times \rho|\cdot|^{x} \rtimes \sigma^{\prime}\right)=2 \cdot \sigma^{\prime},
$$

we have $\operatorname{Jac}_{\rho|\cdot|{ }^{x+1}, \rho|\cdot|^{x}, \rho|\cdot|^{x}}(\pi(\phi, \eta)) \subset 2 \cdot \sigma^{\prime}$, as desired.

Suppose that $x>0$. Let $\phi=\phi_{0} \oplus\left(\rho \otimes S_{2 x+1}\right)^{\oplus 2}$ with $\rho \otimes S_{2 x+1} \not \subset \phi_{0}$, and $\eta \in \widehat{\mathcal{A}_{\phi}}$.

Lemma 5.4. Set $\phi_{1}=\phi-\left(\rho \otimes S_{2 x+1}\right)^{\oplus 2} \oplus\left(\rho \otimes S_{2 x-1}\right)^{\oplus 2}$. We canonically identify $\mathcal{A}_{\phi_{1}}$ with $\mathcal{A}_{\phi}$, and let $\eta_{1} \in \widehat{\mathcal{A}_{\phi_{1}}}$ be the character corresponding to $\eta \in \widehat{\mathcal{A}_{\phi}}$. Then we have

$$
\operatorname{Jac}_{\left.\rho|\cdot|\right|^{x}, \rho|\cdot|{ }^{x}}(\pi(\phi, \eta))=2 \cdot \pi\left(\phi_{1}, \eta_{1}\right) .
$$

Proof. Let $\eta_{+}=\eta$ and $\eta_{-} \in \widehat{\mathcal{A}_{\phi}}$ be as in the statement of Theorem 4.2 (3). We also take $\eta_{1, \pm} \in \widehat{\mathcal{A}_{\phi_{1}}}$ corresponding to $\eta_{ \pm}$. Then we have

- $\pi\left(\phi, \eta_{+}\right) \oplus \pi\left(\phi, \eta_{-}\right)=\operatorname{St}(\rho, 2 x+1) \rtimes \pi\left(\phi_{0}, \eta \mid \mathcal{A}_{\phi_{0}}\right)$;

- $\pi\left(\phi_{1}, \eta_{1,+}\right) \oplus \pi\left(\phi_{1}, \eta_{1,-}\right)=\operatorname{St}(\rho, 2 x-1) \rtimes \pi\left(\phi_{0}, \eta \mid \mathcal{A}_{\phi_{0}}\right)$;

- $\operatorname{Jac}_{\rho|\cdot|^{x}, \rho|\cdot| x}\left(\operatorname{St}(\rho, 2 x+1) \rtimes \pi\left(\phi_{0}, \eta \mid \mathcal{A}_{\phi_{0}}\right)\right) \cong 2 \cdot \operatorname{St}(\rho, 2 x-1) \rtimes \pi\left(\phi_{0}, \eta \mid \mathcal{A}_{\phi_{0}}\right)$.

Therefore, it is enough to show that $\operatorname{Jac}_{\rho|\cdot|^{x}, \rho|\cdot| x}(\pi(\phi, \eta)) \subset 2 \cdot \pi\left(\phi_{1}, \eta_{1}\right)$.

We apply Møglin's construction to $\Pi_{\phi}$. Write

$$
\phi=\left(\bigoplus_{i=1}^{t} \rho \otimes S_{a_{i}}\right) \oplus \phi_{e}
$$


with $a_{1} \leq \cdots \leq a_{t}$ and $\rho \otimes S_{a} \not \subset \phi_{e}$ for any $a>0$. There exists $t_{0}>1$ such that $a_{t_{0}-1}=a_{t_{0}}=$ $2 x+1$. Take a new $L$-parameter

$$
\phi_{\gg}=\left(\bigoplus_{i=1}^{t} \rho \otimes S_{a_{i}^{\prime}}\right) \oplus \phi_{e}
$$

such that

- $a_{1}^{\prime}<\cdots<a_{t}^{\prime}$

- $a_{i}^{\prime} \geq a_{i}$ and $a_{i}^{\prime} \equiv a_{i} \bmod 2$ for any $i$.

In particular, $a_{t_{0}}^{\prime} \geq 2 x+3$. We can identify $\mathcal{A}_{\phi \gg}$ with $\mathcal{A}_{\phi}$ canonically. Let $\eta_{\gg} \in \widehat{\mathcal{A}_{\phi_{\gg}}}$ be the character corresponding to $\eta \in \widehat{\mathcal{A}_{\phi}}$. Then Theorem 3.3 says that

$$
\pi(\phi, \eta)=\mathrm{Jac}_{\rho|\cdot| \frac{a_{t}^{\prime}-1}{2}, \ldots, \rho|\cdot|^{\frac{a_{t}+1}{2}}} \circ \cdots \circ \mathrm{Jac}_{\rho|\cdot| \frac{a_{1}^{\prime}-1}{2}}, \ldots, \rho|\cdot| \frac{a_{1}+1}{2}\left(\pi\left(\phi_{\gg}, \eta_{\gg}\right)\right) .
$$

Note that $\left(a_{t_{0}}+1\right) / 2=x+1$. By Lemma 2.5 (2), we see that

$$
\begin{aligned}
& \operatorname{Jac}_{\rho|\cdot| x, \rho|\cdot| x}(\pi(\phi, \eta))
\end{aligned}
$$

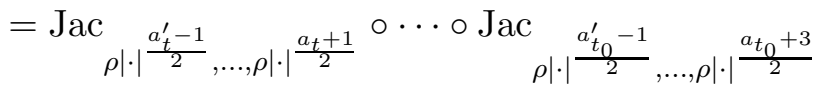

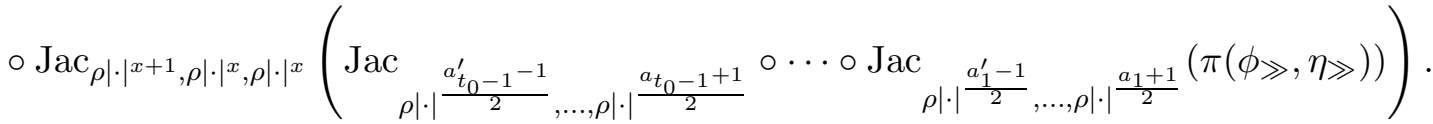

By Lemma 5.3, we have

$$
\begin{aligned}
& \operatorname{Jac}_{\rho|\cdot| x+1, \rho|\cdot| x, \rho|\cdot| x}\left(\operatorname{Jac}_{\rho|\cdot| \frac{a_{t_{0}-1}^{\prime}-1}{2}, \ldots, \rho|\cdot|}{\frac{a_{t_{0}-1}+1}{2}} \cdots \operatorname{Jac}_{\rho|\cdot| \frac{a_{1}^{\prime}-1}{2}, \ldots, \rho|\cdot| \frac{a_{1}+1}{2}}\left(\pi\left(\phi_{\gg,}, \eta_{\gg}\right)\right)\right)
\end{aligned}
$$

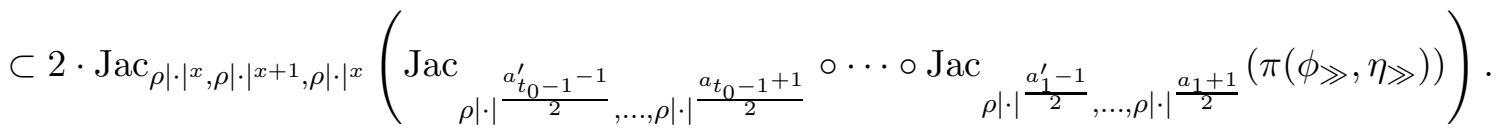

Since

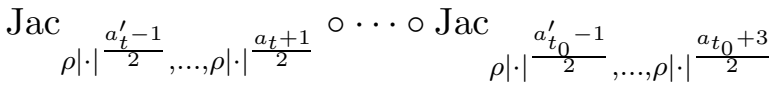

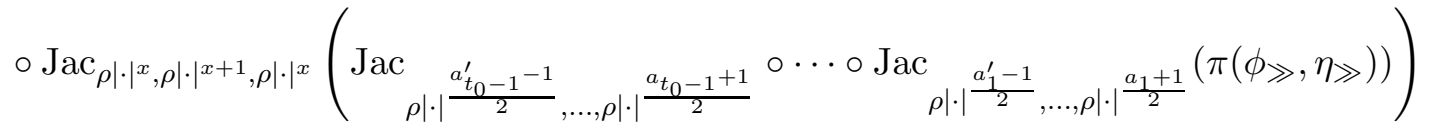

$$
\begin{aligned}
& =\pi\left(\phi_{1}, \eta_{1}\right)
\end{aligned}
$$

by Theorem 3.3, we have $\operatorname{Jac}_{\rho|\cdot| x, \rho|\cdot| x}(\pi(\phi, \eta)) \subset 2 \cdot \pi\left(\phi_{1}, \eta_{1}\right)$, as desired.

Now we can prove Theorem 4.2 (3).

Proof of Theorem 4.2 (3). By Corollary 2.8, we have

$$
\operatorname{Jac}_{\rho|\cdot| x}\left(\pi\left(\phi, \eta_{+}\right) \oplus \pi\left(\phi, \eta_{-}\right)\right)=2 \cdot\langle\rho ; x, x-1, \ldots,-(x-1)\rangle \rtimes \pi\left(\phi_{0}, \eta \mid \mathcal{A}_{\phi_{0}}\right) .
$$

By Proposition [5.2, we have an exact sequence

$$
0 \longrightarrow \pi\left(\phi^{\prime}, \eta_{+}^{\prime}\right) \oplus \pi\left(\phi^{\prime}, \eta_{-}^{\prime}\right) \longrightarrow \Pi \longrightarrow \sigma \longrightarrow 0,
$$


where $\Pi=\langle\rho ; x, x-1, \ldots,-(x-1)\rangle \rtimes \pi\left(\phi_{0}, \eta \mid \mathcal{A}_{\phi_{0}}\right)$, and $\sigma$ is the unique irreducible quotient of П. Fix $\epsilon \in\{ \pm 1\}$. By Lemma 5.4, we see that $\operatorname{Jac}_{\rho|\cdot| x}\left(\pi\left(\phi, \eta_{\epsilon}\right)\right) \supset 2 \cdot \pi\left(\phi^{\prime}, \eta_{\epsilon}^{\prime}\right)$. On the other hand, since s.s. $\operatorname{Jac}_{P_{d(2 x+1)}}\left(\pi\left(\phi, \eta_{\epsilon}\right)\right) \supset \operatorname{St}(\rho, 2 x+1) \otimes \pi\left(\phi_{0}, \eta \mid \mathcal{A}_{\phi_{0}}\right)$, we have

$$
\text { s.s.Jac } \operatorname{Jac}_{P_{2 d x}}\left(\operatorname{Jac}_{\rho|\cdot| x}\left(\pi\left(\phi, \eta_{\epsilon}\right)\right)\right) \supset|\cdot|^{-\frac{1}{2}} \operatorname{St}(\rho, 2 x) \otimes \pi\left(\phi_{0}, \eta \mid \mathcal{A}_{\phi_{0}}\right) .
$$

This implies that $\mathrm{Jac}_{\rho|\cdot| x}\left(\pi\left(\phi, \eta_{\epsilon}\right)\right)$ contains an irreducible non-tempered representation, which must be $\sigma$. Hence

$$
\operatorname{Jac}_{\rho|\cdot| x}\left(\pi\left(\phi, \eta_{\epsilon}\right)\right) \supset 2 \cdot \pi\left(\phi^{\prime}, \eta_{\epsilon}\right)+\sigma=\Pi+\pi\left(\phi^{\prime}, \eta_{\epsilon}\right)-\pi\left(\phi^{\prime}, \eta_{-\epsilon}\right) .
$$

Considering $\operatorname{Jac}_{\rho|\cdot| x}\left(\pi\left(\phi, \eta_{+}\right) \oplus \pi\left(\phi, \eta_{-}\right)\right)$, we see that this inclusion must be an equality.

If $x=0$ and $m=2$, we see that $\operatorname{Jac}_{\rho}(\pi(\phi, \eta)) \supset \pi\left(\phi_{0}, \eta \mid \mathcal{A}_{\phi_{0}}\right)$. By the same argument, this inclusion must be an equality. This completes the proof of Theorem 4.2 (4), so that the ones of all statements of Theorem 4.2 .

5.5. Description of $\mu_{\rho}^{*}$. We prove Theorem 4.3 in this subsection. To do this, we need the following specious lemma.

Lemma 5.5. Let $\phi \in \Phi_{\mathrm{gp}}(G)$ and $\underline{x}=\left(x_{1}, \ldots, x_{m}\right) \in \mathbb{R}^{m}$. Suppose that $\operatorname{Jac}_{\rho|\cdot| \underline{x}}(\pi) \neq 0$ for some $\pi \in \Pi_{\phi}$.

(1) If $x_{m}<0$, then $x_{i}=-x_{m}$ for some $i$.

(2) Suppose that $\underline{x}$ is of the form

$$
\underline{x}=(\underbrace{x_{1}^{(1)}, \ldots, x_{m_{1}}^{(1)}}_{m_{1}}, \ldots, \underbrace{x_{1}^{(k)}, \ldots, x_{m_{k}}^{(k)}}_{m_{k}})
$$

with $x_{i-1}^{(j)}>x_{i}^{(j)}$ for $1 \leq j \leq k, 1<i \leq m_{j}$, and $x_{1}^{(1)} \leq \cdots \leq x_{1}^{(k)}$. Then $x_{1}^{(j)} \geq 0$ for $j=1, \ldots, k$, and

$$
\phi \supset \bigoplus_{j=1}^{k} \rho \otimes S_{2 x_{1}^{(j)}+1} .
$$

Proof. We prove the lemma by induction on $m$. By Lemma 4.1, we see that $2 x_{1}+1$ is a positive integer, and $\phi$ contains $\rho \otimes S_{2 x_{1}+1}$. In particular, we obtain the lemma for $m=1$.

Suppose that $m \geq 2$ and put $\underline{x}^{\prime}=\left(x_{2}, \ldots, x_{m}\right) \in \mathbb{R}^{m-1}$. By Theorem 4.2, one of the following holds.

- $\operatorname{Jac}_{\rho|\cdot| \underline{x^{\prime}}}\left(\pi^{\prime}\right) \neq 0$ for some $\pi^{\prime} \in \Pi_{\phi^{\prime}}$ with $\phi^{\prime}=\phi-\left(\rho \otimes S_{2 x_{1}+1}\right) \oplus\left(\rho \otimes S_{2 x_{1}-1}\right)$;

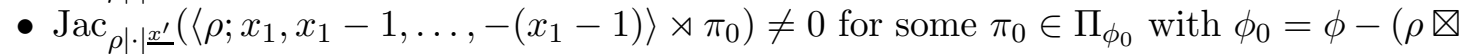
$\left.S_{2 x_{1}+1}\right)^{\oplus 2}$.

The former case can occur only if $x_{1}>0$, and the latter case can occur only if $\phi \supset(\rho \bigotimes$ $\left.S_{2 x_{1}+1}\right)^{\oplus 2}$.

We consider the former case. Assume that $x_{1}>0$ and $\mathrm{Jac}_{\rho|\cdot| \underline{\underline{x^{\prime}}}}\left(\pi^{\prime}\right) \neq 0$ for some $\pi^{\prime} \in \Pi_{\phi^{\prime}}$ with $\phi^{\prime}=\phi-\left(\rho \otimes S_{2 x_{1}+1}\right) \oplus\left(\rho \otimes S_{2 x_{1}-1}\right)$. By the induction hypothesis, we have $x_{i}=-x_{m}$ for some $i \geq 2$ when $x_{m}<0$, and

$$
\phi^{\prime} \supset\left(\rho \otimes S_{2 x_{1}^{(1)}-1}\right) \oplus\left(\bigoplus_{j=2}^{k} \rho \otimes S_{2 x_{1}^{(j)}+1}\right)
$$


when $\underline{x}$ is of the form in (2) since $\underline{x^{\prime}}$ is also of the form. This implies the assertion for $\phi$.

We consider the latter case. Assume that $\phi \supset\left(\rho \otimes S_{2 x_{1}+1}\right)^{\oplus 2}$, and that

$$
\mathrm{Jac}_{\rho|\cdot| \underline{\underline{x^{\prime}}}}\left(\left\langle\rho ; x_{1}, x_{1}-1, \ldots,-\left(x_{1}-1\right)\right\rangle \rtimes \pi_{0}\right) \neq 0
$$

for some $\pi_{0} \in \Pi_{\phi_{0}}$ with $\phi_{0}=\phi-\left(\rho \otimes S_{2 x_{1}+1}\right)^{\oplus 2}$. By Corollary 2.8, we can divide

$$
\{2, \ldots, m\}=\left\{i_{1}, \ldots, i_{m_{1}}\right\} \sqcup\left\{j_{1}, \ldots, j_{m_{2}}\right\} \sqcup\left\{k_{1}, \ldots, k_{m_{3}}\right\}
$$

with $i_{1}<\cdots<i_{m_{1}}, j_{1}<\cdots<j_{m_{2}}, k_{1}<\cdots<k_{m_{3}}$ and $m_{2}+m_{3} \leq 2 x_{1}$ such that

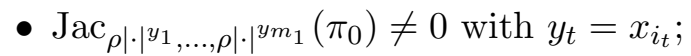

- $x_{j_{t}}=x_{1}+1-t$ for $t=1, \ldots, m_{2}$;

- $x_{k_{t}}=x_{1}-t$ for $t=1, \ldots, m_{3}$.

Considering the following four cases, we can prove the existence $x_{i}$ satisfying $x_{i}=-x_{m}$ when $x_{m}<0$.

- When $m=i_{m_{1}}$, by the induction hypothesis, we have $x_{i_{t}}=-x_{m}$ for some $t$.

- When $m=j_{m_{2}}$, we have $x_{m}=x_{1}+1-m_{2}<0$ so that $x_{j_{t}}=-x_{m}$ with $t=2 x_{1}+2-m_{2}$.

- When $m=k_{m_{3}}$ and $m_{3}<2 x_{1}$, we have $x_{m}=x_{1}-m_{3}<0$ so that $x_{k_{t}}=-x_{m}$ with $t=2 x_{1}-m_{3}$.

- When $m=k_{m_{3}}$ and $m_{3}=2 x_{1}$, we have $x_{1}=-x_{m}$.

On the other hand, when $\underline{x}$ is of the form in (2), since $x_{1}^{(j)} \geq x_{1}^{(1)}=x_{1}$, there is at most one $j_{0} \geq 2$ such that

$$
x_{1}^{\left(j_{0}\right)} \in\left\{x_{j_{t}} \mid t=1, \ldots, m_{2}\right\} \cup\left\{x_{k_{t}} \mid t=1, \ldots, m_{3}\right\},
$$

in which case, $x_{1}^{\left(j_{0}\right)}=x_{1}$. By the induction hypothesis, we have

$$
\phi_{0} \supset \bigoplus_{\substack{2 \leq j \leq k \\ j \neq j}} \rho \otimes S_{2 x_{1}^{(j)}+1} .
$$

This implies the assertion for $\phi$. This completes the proof.

Now we can prove Theorem 4.3 .

Proof of Theorem 4.3. Since the subgroup of $\mathcal{R}_{m}$ spanned by $\operatorname{Irr}_{\rho}\left(\mathrm{GL}_{d m}(F)\right)=\left\{\tau_{\underline{x}} \mid \underline{x} \in \Omega_{m}\right\}$ has another basis $\left\{\Delta_{\underline{x}} \mid \underline{x} \in \Omega_{m}\right\}$, we can write

$$
\mu_{\rho}^{*}(\pi)=\sum_{m \geq 0} \sum_{\underline{x} \in \Omega_{m}} \Delta_{\underline{x}} \otimes \Pi_{\underline{x}}(\pi)
$$

for some virtual representation $\Pi_{\underline{x}}(\pi)$. For $\underline{y} \in \Omega_{m}$, applying $\operatorname{Jac}_{\rho|\cdot| \underline{y}}$ to s.s. $\operatorname{Jac}_{P_{d m}}(\pi)$, we have

$$
\operatorname{Jac}_{\rho|\cdot| \underline{y}}(\pi)=\sum_{\underline{x} \in \Omega_{m}} \operatorname{Jac}_{\rho|\cdot| \underline{y}}\left(\Delta_{\underline{x}}\right) \otimes \Pi_{\underline{x}}(\pi)=\sum_{\underline{x} \in \Omega_{m}} m(\underline{y}, \underline{x}) \cdot \Pi_{\underline{x}}(\pi),
$$

where $m(\underline{y}, \underline{x})=\operatorname{dim}_{\mathbb{C}} \operatorname{Jac}_{\rho|\cdot| \underline{y}}\left(\Delta_{\underline{x}}\right)$. If $m^{\prime}\left(\underline{x^{\prime}}, \underline{y}\right) \in \mathbb{Q}$ satisfies that

$$
\sum_{\underline{y} \in \Omega_{m}} m^{\prime}\left(\underline{x^{\prime}}, \underline{y}\right) m(\underline{y}, \underline{x})=\delta_{\underline{x^{\prime}}, \underline{x}}
$$

we have

$$
\Pi_{\underline{x}}(\pi)=\sum_{\underline{y} \in \Omega_{m}} m^{\prime}(\underline{x}, \underline{y}) \cdot \operatorname{Jac}_{\rho|\cdot| \underline{y}}(\pi) .
$$


Hence we have

$$
\mu_{\rho}^{*}(\pi)=\sum_{m \geq 0} \sum_{\underline{x}, \underline{y} \in \Omega_{m}} m^{\prime}(\underline{x}, \underline{y}) \cdot \Delta_{\underline{x}} \otimes \operatorname{Jac}_{\rho|\cdot| \underline{y}}(\pi) .
$$

One can easily to prove by induction that $m^{\prime}(\underline{x}, \underline{x}(\underline{k}))=0$ unless $\underline{x}=\underline{x}\left(\underline{k^{\prime}}\right)$ for some $\underline{k^{\prime}} \in K_{\phi}$ (see also the proof of Lemma 2.4). Therefore, by Lemma 5.5, we have

$$
\mu_{\rho}^{*}(\pi)=\sum_{m \geq 0} \sum_{\underline{k}, \underline{k^{\prime}} \in K_{\phi}} m^{\prime}\left(\underline{x}\left(\underline{k^{\prime}}\right), \underline{x}(\underline{k})\right) \cdot \Delta_{\underline{x}\left(\underline{k^{\prime}}\right)} \otimes \operatorname{Jac}_{\rho|\cdot| \underline{x} \underline{k})}(\pi) .
$$

This completes the proof of Theorem 4.3 .

5.6. A remark on standard modules. As a consequence of Theorem 4.2, we can prove the irreducibility of certain standard modules.

Corollary 5.6. Let $\phi \in \Phi_{\mathrm{gp}}(G)$ and $\eta \in \widehat{\mathcal{A}_{\phi}}$ such that $\pi(\phi, \eta) \neq 0$. Suppose that $\phi \supset \rho \bigotimes S_{2 x+1}$ for $x>0$ but $\operatorname{Jac}_{\rho|\cdot| x}(\pi(\phi, \eta))=0$. Then the standard module

$$
\Pi=\langle\rho ; x, x-1, \ldots,-(x-1)\rangle \rtimes \pi(\phi, \eta)
$$

is irreducible.

Proof. Let $\sigma$ be the unique irreducible quotient of $\Pi$, which is non-tempered. By the same argument as the proof of Proposition 5.2, we see that $\sigma$ is the unique irreducible non-tempered subquotient of $\Pi$. Suppose that $\Pi$ is reducible. If $\pi^{\prime}$ is another irreducible subquotient of $\Pi$, by considering its cuspidal support or its Plancherel measure, we see that $\pi^{\prime} \in \Pi_{\phi^{\prime}}$ with $\phi^{\prime}=$ $\phi \oplus\left(\rho \bigotimes S_{2 x-1}\right) \oplus\left(\rho \bigotimes S_{2 x+1}\right)$. Since $\phi \supset \rho \bigotimes S_{2 x+1}$, we see that $\phi^{\prime} \supset\left(\rho \bigotimes S_{2 x+1}\right)^{\oplus 2}$. By Theorem 4.2, $\mathrm{Jac}_{\rho|\cdot| x}\left(\pi^{\prime}\right)$ contains an irreducible non-tempered representation. However, $\operatorname{Jac}_{\rho|\cdot|{ }^{x}}(\Pi)=$ $\operatorname{St}(\rho, 2 x-1) \rtimes \pi(\phi, \eta)$ consists of tempered representations. This is a contradiction.

Example 5.7. Consider $\phi=S_{2} \oplus S_{4} \oplus S_{6} \in \Phi_{\text {disc }}\left(\mathrm{SO}_{13}\right)$ and $\eta \in \widehat{A_{\phi}}$ given by $\eta\left(\alpha_{S_{2 a}}\right)=(-1)^{a}$ for $a=1,2,3$. Then $\pi(\phi, \eta)$ is an irreducible supercuspidal representation. Moreover, the standard module

$$
\Pi=\left\langle\mathbf{1}_{\mathrm{GL}_{1}(F)} ; \frac{5}{2}, \frac{3}{2}, \frac{1}{2},-\frac{1}{2},-\frac{3}{2}\right\rangle \rtimes \pi(\phi, \eta)
$$

of $\mathrm{SO}_{23}(F)$ is irreducible. Note that the L-parameter $\phi^{\prime}=\phi \oplus|\cdot|^{\frac{1}{2}} S_{5} \oplus|\cdot|^{-\frac{1}{2}} S_{5}$ of $\Pi$ is non-generic since

$$
L\left(s, \phi^{\prime}, \mathrm{Ad}\right)=\zeta_{F}(s-1) \zeta_{F}(s)^{3} \zeta_{F}(s+1)^{13} \zeta_{F}(s+2)^{10} \zeta_{F}(s+3)^{12} \zeta_{F}(s+4)^{5} \zeta_{F}(s+5)^{3}
$$

has a pole at $s=1$, where $\zeta_{F}$ is the local zeta function associated to $F$. In particular, the standard module

$$
\Pi_{0}=\left\langle\mathbf{1}_{\mathrm{GL}_{1}(F)} ; \frac{5}{2}, \frac{3}{2}, \frac{1}{2},-\frac{1}{2},-\frac{3}{2}\right\rangle \rtimes \pi(\phi, \mathbf{1})
$$

is reducible by Theorem 3.2. 
HIRAKU ATOBE

\section{REFERENCES}

[Ar13] J. Arthur, The endoscopic classification of representations. Orthogonal and symplectic groups. American Mathematical Society Colloquium Publications, 61. American Mathematical Society, Providence, RI, 2013.

[AMS18] A.-M. Aubert, A. Moussaoui and M. Solleveld, Generalizations of the Springer correspondence and cuspidal Langlands parameters. Manuscripta Math. 157 (2018), no. 1-2, 121-192.

[AMSa] A.-M. Aubert, A. Moussaoui and M. Solleveld, Graded Hecke algebras for disconnected reductive group. arXiv:1607.02713v2.

[AMSb] A.-M. Aubert, A. Moussaoui and M. Solleveld, Affine Hecke algebras for Langlands parameters. arXiv:1701.03593 2 .

[CS98] W. Casselman and F. Shahidi, On irreducibility of standard modules for generic representations. Ann. Sci. École Norm. Sup. (4) 31 (1998), no. 4, 561-589.

[GI16] W. T. Gan and A. Ichino, The Gross-Prasad conjecture and local theta correspondence. Invent. Math. 206 (2016), no. 3, 705-799.

[HM07] V. Heiermann and G. Muić, On the standard modules conjecture. Math. Z. 255 (2007), no. 4, 847-853.

[HO13] V. Heiermann and E. Opdam, On the tempered L-functions conjecture. Amer. J. Math. 135 (2013), no. 3, 777-799.

[H16] V. Heiermann, , A note on standard modules and Vogan L-packets. Manuscripta Math. 150 (2016), no. $3-4,571-583$.

[M06] C. Mœglin, Sur certains paquets d'Arthur et involution d'Aubert-Schneider-Stuhler généralisée. Represent. Theory 10 (2006), 86-129.

[M09] C. Møglin, Paquets d'Arthur discrets pour un groupe classique p-adique. Automorphic forms and Lfunctions II. Local aspects, 179-257, Contemp. Math., 489, Israel Math. Conf. Proc., Amer. Math. Soc., Providence, RI, 2009.

[MW12] C. Møglin and J.-L. Waldspurger, La conjecture locale de Gross-Prasad pour les groupes spéciaux orthogonaux: le cas général. Sur les conjectures de Gross et Prasad. II. Astérisque No. 347 (2012), 167216.

[Mu01] G. Muić, A proof of Casselman-Shahidi's conjecture for quasi-split classical groups. Canad. Math. Bull. 44 (2001), no. 3, 298-312.

[T95] M. Tadić, Structure arising from induction and Jacquet modules of representations of classical p-adic groups, J. Algebra 177 (1995), no. 1, 1-33.

[X17a] B. Xu, On the cuspidal support of discrete series for p-adic quasisplit $\mathrm{Sp}(N)$ and $\mathrm{SO}(N)$. Manuscripta Math. 154 (2017), no. 3-4, 441-502.

[X17b] B. Xu, On Mœglin's parametrization of Arthur packets for p-adic quasisplit $\operatorname{Sp}(N)$ and $\operatorname{SO}(N)$. Canad. J. Math. 69 (2017), no. 4, 890-960.

[Z80] A. V. Zelevinsky, Induced representations of reductive $\mathfrak{p}$-adic groups. II. On irreducible representations of GL(n), Ann. Sci. École Norm. Sup. (4) 13 (1980), no. 2, 165-210.

Department of Mathematics, Hokkaido University Kita 10, Nishi 8, Kita-Ku, Sapporo, Hokkaido, 060-0810, JAPAN

E-mail address: atobe@math.sci.hokudai.ac.jp 